Fermilab-Pub-97/164-T

KA-TP-9-1997

May 1997

\title{
Top Pair Production at Hadron Colliders in non-minimal Standard Models
}

\author{
W. Hollik ${ }^{a}$, W.M. Mösle ${ }^{a}$ and D. Wackeroth ${ }^{b *}$ \\ ${ }^{a}$ Institut für Theoretische Physik, Universität Karlsruhe, D-76128 Karlsruhe, Germany \\ ${ }^{b}$ Theory Group, Fermi National Accelerator Laboratory, Batavia, IL 60510, USA
}

\begin{abstract}
The cross section of top pair production in hadronic collisions to $\mathcal{O}\left(\alpha \alpha_{s}^{2}\right)$ is calculated within the General 2-Higgs-Doublet Model and the Minimal Supersymmetric Standard Model. At the parton level the $\mathcal{O}(\alpha)$ one-loop corrections to the main production mechanisms, $q \bar{q} \rightarrow t \bar{t}$ and $g g \rightarrow t \bar{t}$, significantly modify the Born-cross sections: in the threshold region $\sqrt{\hat{s}} \gtrsim 2 m_{t}$ they are enhanced up to $50 \%$ and with increasing $\mathrm{cm}$ energy $\sqrt{\hat{s}}$, they can be reduced by up to the same order of magnitude. In a wide range of the parameter space of the models under consideration the observable hadronic cross sections for top pair production at the Tevatron $p \bar{p} \rightarrow t \bar{t} X$ and at the LHC $p p \rightarrow t \bar{t} X$ are typically reduced by several percent $(\lesssim 10 \%)$ compared to the lowest order result. In special regions of the parameter space, that is in the vicinity of the threshold for the top quark decay $t \rightarrow b H^{+} ; \tilde{t} \tilde{\chi}^{0}$, the radiative corrections are considerably enhanced, comparable in size to QCD effects.
\end{abstract}

*E-mail: dow@fnth09.fnal.gov 


\section{INTRODUCTION}

The discovery of the top quark in 1995 by the CDF [1] and D $\varnothing$ [2] experiments at the Fermilab Tevatron once again impressively confirmed the Minimal Standard Model (MSM) [3, 团] as a valid description of electroweak particle interactions up to presently accessible energies. In the meantime, experimental effort has been concentrated on detailed studies of the top quark properties. Presently, the top quark mass is known to be (world average) [5]

$$
m_{t}=175.6 \pm 5.5 \mathrm{GeV}
$$

which is consistent with the MSM prediction obtained by performing a global MSM-fit to all available electroweak precision data [6]

$$
m_{t}=172.7 \pm 5.4 \mathrm{GeV} .
$$

The measurement of the top pair production cross section $\sigma_{t \bar{t}}$ at hadron colliders is a significant test of the Standard Model. The observation of deviations from the Standard Model predictions, including electroweak and QCD corrections, could indicate new non-standard production or decay mechanisms. The measurement of $\sigma_{t \bar{t}}$ performed at the Tevatron [0,5]

$$
\begin{aligned}
\mathrm{CDF} & : \sigma_{t \bar{t}}\left(m_{t}=175 \mathrm{GeV}\right)=7.5_{-1.6}^{+1.9} \mathrm{pb} \\
\mathrm{D} \varnothing: \sigma_{t \bar{t}}\left(m_{t}=170 \mathrm{GeV}\right) & =5.77 \pm 1.76 \mathrm{pb}
\end{aligned}
$$

is largely in good agreement with the theoretical QCD prediction [8 10]

$$
\begin{gathered}
\sigma_{t \bar{t}}\left(m_{t}=175 \mathrm{GeV}\right)=5.52_{-0.42}^{+0.07} \mathrm{pb} \quad, \sigma_{t \bar{t}}\left(m_{t}=170 \mathrm{GeV}\right)=6.48_{-0.48}^{+0.09} \mathrm{pb} \\
\sigma_{t \bar{t}}\left(m_{t}=175 \mathrm{GeV}\right)=4.75_{-0.68}^{+0.63} \mathrm{pb} \\
\sigma_{t \bar{t}}\left(m_{t}=170 \mathrm{GeV}\right)=5.83_{-0.51}^{+0.85} \mathrm{pb} .
\end{gathered}
$$

The complete MSM electroweak one-loop corrections to top pair production at hadron colliders are calculated as well [11] but have only little impact on $\sigma_{t \bar{t}}$ at the Tevatron $(\sim 1 \%)$. The value of $\sigma_{t \bar{t}}$ measured by the CDF collaboration is slightly higher than the theoretical prediction but the study of systematic uncertainties and the combination of different decay channels is still in progress [7]. The current large experimental uncertainty, however, still leaves room for non-standard physics effects through the virtual presence of new particles, for instance. At the upgraded Tevatron with $\mathcal{L}=10 \mathrm{fb}^{-1}$ a measurement of $m_{t}$ and of the total $t \bar{t}$ production rate with a precision of $\delta m_{t}=2 \mathrm{GeV}$ and of $\delta \sigma_{t \bar{t}} / \sigma_{t \bar{t}}=6 \%$, respectively, is within reach [12]. At the LHC the mass measurement is expected to be more accurate due to higher statistics and the cross section measurement will at least be as precise as that performed at the Tevatron since the Tevatron-estimate is already to a large extent limited by systematic uncertainties [12].

The envisaged high precisions open a new rich field of top quark phenomenology. Here we are going to concentrate on the implications of non-standard electroweak-like radiative corrections, for the top pair production cross section in hadronic collisions.

Since the mechanism which introduces gauge boson masses in a gauge invariant way by spontaneously breaking the electroweak symmetry [4] is the least experimentally explored sector of the MSM, possible extensions of the Higgs-sector are of particular interest. There, the consideration of a second Higgs-doublet plays a special role [13]: 
- the extension to two Higgs-doublets represents a minimal extension of the MSM Higgssector as far as the number of newly introduced parameters is concerned,

- as a new physical phenomenon, charged Higgs-bosons occur,

- the requirement $\rho=\frac{M_{W}^{2}}{c_{W}^{2} M_{Z}^{2}}=1$ at Born-level is still fulfilled and

- two Higgs-doublets are required in a minimal supersymmetric extension of the MSM.

Supersymmetry (SUSY) 14 represents an additional symmetry between fermions and bosons which implemented in the MSM solves such MSM deficiencies like the hierarchy problem, the necessity of fine tuning and the non-occurrence of gauge coupling unification at high energies.

Past studies of non-minimal Standard Model implications on the top pair production cross section at hadron colliders comprised the calculation of

- the $\mathcal{O}(\alpha)$ one-loop corrections within the General 2-Higgs-Doublet Model (G2HDM) to both the $q \bar{q}$ annihilation and gluon fusion subprocesses with numerical results for the Tevatron [16] and the LHC [17, 18],

- the SUSY QCD $\mathcal{O}\left(\alpha_{s}\right)$ contribution to the $q \bar{q}$ annihilation and gluon fusion subprocesses with numerical results for the Tevatron [19,20] and the LHC [21], and

- the SUSY electroweak-like (EW-like) one-loop corrections to the $q \bar{q}$ annihilation subprocess with numerical results for the Tevatron [20,22].

The electroweak radiative corrections are of special interest due to the strong Yukawacouplings of the top quark to the Higgs-bosons. While the MSM prediction of the electroweak one-loop contribution does not exceed $\sim 3 \%$ of the Born-cross section [11,15] the models discussed here involve the interesting possibility of an enhancement of the Yukawa-couplings and of additional contributions through the virtual presence of supersymmetric particles. Thus, we give a complete description of the top pair production cross sections to $\mathcal{O}\left(\alpha \alpha_{s}^{2}\right)$ of both main production mechanisms, $q \bar{q}$ annihilation and gluon fusion, within the G2HDM (= Standard Model with two Higgs-doublets but without imposing SUSY constraints) and the Minimal Supersymmetric Standard Model (MSSM) which involves the following one-loop contributions:

- the electroweak gauge boson contributions ( $W$ and $Z$ boson exchange),

- the Higgs-boson contributions within the G2HDM,

- the EW-like MSSM contribution, where the contribution of the supersymmetric Higgssector and the SUSY EW-like one-loop corrections are discussed separately.

We provide explicit analytical expressions for the form factors which parametrize the oneloop modifications of the $g t \bar{t}$-vertex, present numerical results at the parton level and give a detailed discussion of their numerical significance at the upgraded Tevatron with $\sqrt{S}=2$ $\mathrm{TeV}$ and at the LHC with $\sqrt{S}=14 \mathrm{TeV}$. 


\section{TOP PAIR PRODUCTION IN NON-MINIMAL STANDARD MODELS}

The main production mechanism for $t \bar{t}$ production at the Tevatron is the annihilation of a quark-antiquark pair

$$
q\left(p_{4}\right)+\bar{q}\left(p_{3}\right) \rightarrow t\left(p_{2}\right)+\bar{t}\left(p_{1}\right)
$$

whereas at the LHC the top quark pairs are mainly produced via the fusion of two gluons

$$
g\left(p_{4}\right)+g\left(p_{3}\right) \rightarrow t\left(p_{2}\right)+\bar{t}\left(p_{1}\right) .
$$

At the parton level, the corresponding differential cross sections to order $\mathcal{O}\left(\alpha \alpha_{s}^{2}\right)$ are obtained by contracting the matrix elements describing the $\mathcal{O}(\alpha)$ contribution to these subprocesses $\delta \mathcal{M}_{i}, i=q \bar{q}, g g$ with the Born-matrix elements $\mathcal{M}_{B}^{i}$

$$
\delta \frac{d \hat{\sigma}_{i}(\hat{t}, \hat{s})}{d \hat{t}}=\frac{1}{16 \pi^{2} \hat{s}} 2 \mathcal{R} e \bar{\sum}\left(\delta \mathcal{M}_{i} \times \mathcal{M}_{B}^{i *}\right),
$$

where $\hat{t}=\left(p_{3}-p_{1}\right)^{2}, \hat{s}=\left(p_{3}+p_{4}\right)^{2}$ are Mandelstam variables. The explicit representations of $\delta \mathcal{M}_{i}$ as well as $\mathcal{M}_{B}^{i}$ are given in [1] (Eqs.(3.34,3.39)), where the $\mathcal{O}(\alpha)$ contribution within the MSM to both production mechanisms $q \bar{q}$ annihilation and gluon fusion has been studied. Throughout this paper we closely follow the notation of [1] and refer to it for more details. Here we study the modification of the form factors introduced in 11] by non-minimal Standard Model $\mathcal{O}(\alpha)$ contributions. In order to reveal the numerical effect of these corrections on the top pair production cross sections and to study the dependence on the parameters of the underlying model we introduce a relative correction $\Delta_{i}$ at the parton level

$$
\hat{\sigma}_{i}(\hat{s})=\hat{\sigma}_{B}^{i}(\hat{s})+\delta \hat{\sigma}_{i}(\hat{s})=\sigma_{B}^{i}\left(1+\Delta_{i}\right)
$$

where we already carried out the $\hat{t}$-integration. To compare with non-standard results we show in Fig. 1 the relative corrections $\Delta_{q \bar{q}}$ and $\Delta_{g g}$ obtained within the MSM. There, the mass of the MSM Higgs-boson $M_{H}$ is assumed to be within bounds resulting from the negative searches at LEP $M_{H} \geq 66 \mathrm{GeV}$ 23] and from theoretical arguments based on unitarity $M_{H} \leq 1 \mathrm{TeV}$ [24]. In the numerical evaluation the electroweak MSM parameters are chosen to be [25, 6, 26]:

$$
m_{t}=175 \mathrm{GeV}, m_{b}=4.7 \mathrm{GeV}, M_{W}=80.356 \mathrm{GeV}, M_{Z}=91.1863 \mathrm{GeV}, \alpha^{-1}=137.035989
$$

In the following we introduce the relevant features of the models under consideration, present the analytical expression for the form factors to $q \bar{q}$ annihilation and gluon fusion and study their numerical impact.

\section{A. The General 2-Higgs-Doublet Model}

The General 2-Higgs-Doublet Model (G2HDM) [13] introduces six additional parameters into the theory: four masses and two mixing angles. In standard notation they read: 

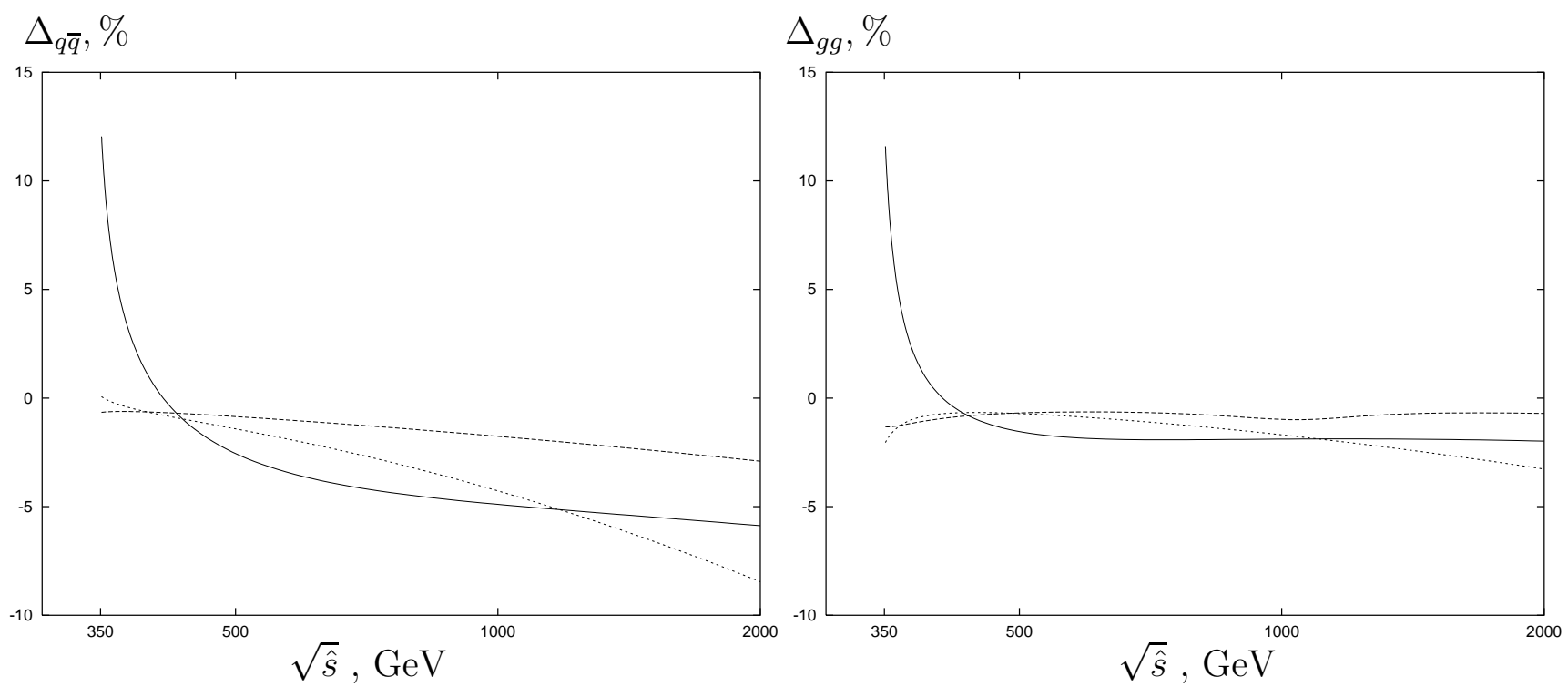

FIG. 1. The relative corrections $\Delta_{q \bar{q}}$ and $\Delta_{g g}$ within the MSM. The $\mathcal{O}(\alpha)$ contribution of the electroweak gauge bosons (dotted line) and of the Higgs-sector are shown separately (solid line: $M_{H}=65 \mathrm{GeV}$, dashed line: $\left.M_{H}=1 \mathrm{TeV}\right)$.

$M_{A^{0}}, M_{h^{0}}, M_{H^{0}}, M_{H^{ \pm}}, \tan \beta$ and $\alpha$. Since we are working in the 't Hooft-Feynman gauge we also have to include the contribution of the Higgs-ghosts $G^{0}$ and $G^{ \pm}$. The G2HDM one-loop corrections to the top pair production processes, $q \bar{q}$ annihilation and gluon fusion, can be easily obtained from [11] when the Yukawa-couplings written in the generalized form

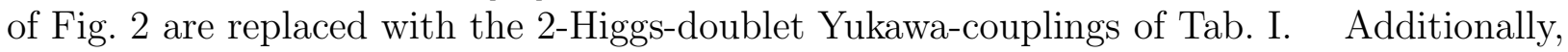
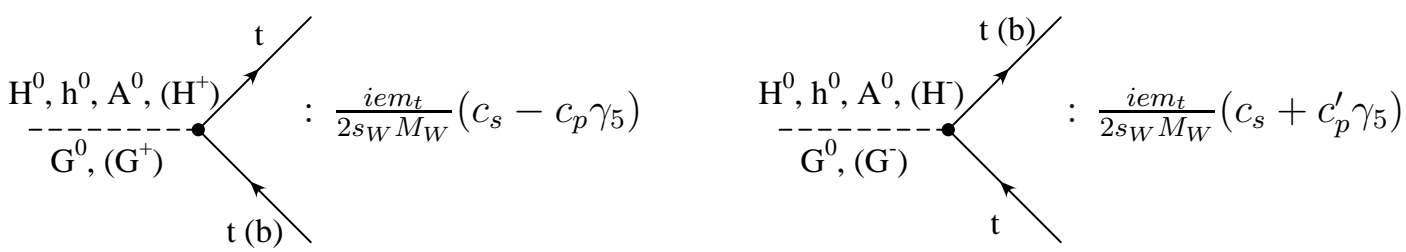

FIG. 2. The Feynman-rules for the $H$-t-t(b)-vertex within the 2-Higgs-Doublet Model written in generalized form. The coupling parameters $c_{s}, c_{p}, c_{p}^{\prime}$ are explicitly given in Tab. I.

the summation over the MSM Higgs-bosons in [11] has to be extended to

$$
\sum_{S=\eta, \chi, \Phi^{ \pm}} \rightarrow \sum_{S=H^{0}, h^{0}, A^{0}, H^{ \pm}, G^{0}, G^{ \pm}}
$$

and now two neutral scalars $H^{0}, h^{0}$ contribute to the $s$-channel Higgs-exchange diagram in the gluon fusion subprocess.

In order to demonstrate the effect of the convolution with parton distribution functions and to study the dependence on the free parameters we present numerical results obtained within the G2HDM also at the parton level. For the numerical evaluation of the electroweak one-loop corrections within the G2HDM we chose the mixing angle $\alpha$ to be $\alpha=\pi / 2$. Then 
TABLE I. The top-Yukawa-couplings within the 2-Higgs-Doublet Model $\left(m_{b}\right.$ : bottom quark mass, $\alpha$ : mixing angle, $\tan \beta$ : ratio of the vacuum expectation values of the two Higgs-doublets).

\begin{tabular}{|ccccccc|}
\hline \hline & $H^{0}$ & $h^{0}$ & $A^{0}$ & $H^{ \pm}$ & $G^{0}$ & $G^{ \pm}$ \\
\hline$c_{s}$ & $-\sin \alpha / \sin \beta$ & $-\cos \alpha / \sin \beta$ & 0 & $\frac{1}{\sqrt{2}}\left(\cot \beta+\frac{m_{b}}{m_{t}} \tan \beta\right)$ & 0 & $\frac{1}{\sqrt{2}}\left(1-\frac{m_{b}}{m_{t}}\right)$ \\
\hline$c_{p}$ & 0 & 0 & $-i \cot \beta$ & $\frac{1}{\sqrt{2}}\left(\cot \beta-\frac{m_{b}}{m_{t}} \tan \beta\right)$ & $-i$ & $\frac{1}{\sqrt{2}}\left(1+\frac{m_{b}}{m_{t}}\right)$ \\
\hline$c_{p}^{\prime}$ & 0 & 0 & $-c_{p}$ & $c_{p}$ & $-c_{p}$ & $c_{p}$ \\
\hline \hline
\end{tabular}

the heavy Higgs-boson $H^{0}$ develops MSM-like Yukawa-couplings for very large values of $\tan \beta$, and $h^{0}$ decouples. For $\alpha=0$ the neutral scalars $H^{0}$ and $h^{0}$ just switch roles and for values in between the sum of their contribution leads to smaller relative corrections. The pseudo scalar $A^{0}$ is expected to contribute noticeably only for small values of $\tan \beta$. It turns out, that there is very little variation $(<2 \%)$ of the relative corrections with $M_{A^{0}}$ for $\tan \beta=0.7$ and no dependence on $M_{A^{0}}$ can be observed for $\tan \beta=70$. Thus, throughout the following numerical discussion within the G2HDM we chose a representative value of $M_{A^{0}}=50 \mathrm{GeV}$. A study of the dependence on the charged Higgs-boson mass $M_{H^{ \pm}}$shows that there is little variation of the partonic cross sections with $M_{H^{ \pm}}$, only for large values of $\tan \beta$ due to the enhancement of the suppression factor $m_{b} / m_{t}$ in the Yukawa-coupling a small dependence can be observed. Thus, in the following discussion we chose a representative value of $M_{H^{ \pm}}=50 \mathrm{GeV}$. The only exception occurs in a very special region in the G2HDM parameter space: $m_{t} \approx M_{H^{ \pm}}+m_{b}$, where due to a discontinuity in the derivative of the $B$ functions the radiative corrections can be considerably enhanced. In Fig. 3 the dependence of the $\mathcal{O}(\alpha)$ Higgs-sector contribution to the $q \bar{q}$ annihilation and gluon fusion subprocesses within the G2HDM on $M_{H^{0}}$ and $\tan \beta$ can be studied. At the parton level, very large corrections up to $+50 \%$ arise in the threshold region $\sqrt{\hat{s}} \approx 2 m_{t}$ assuming $H^{0}$ is very light and $\tan \beta$ very small. As expected due to the structure of the Yukawa-couplings, the relative corrections decrease for increasing values of $\tan \beta$. The increase of the relative corrections for very large $\tan \beta$ is due to the charged Higgs-boson contribution as discussed earlier. In the case of gluon fusion a Breit-Wigner-resonance structure can be observed originating from the $s$-channel Higgs-exchange diagrams when $\sqrt{\hat{s}} \approx M_{H^{0}, h^{0}}>2 m_{t}$.

\section{B. The Higgs-sector of the MSSM}

The requirements of supersymmetry [14, 30] lead to the existence of (at least) one additional Higgs-doublet where the parameters of the Higgs-potential are tightly correlated. At one-loop level, this translates into the improved physical masses and the effective mixing angle $\alpha_{e f f}$ as follows [27:

$$
\begin{aligned}
M_{H^{ \pm}}^{2} & =M_{W}^{2}+M_{A^{0}}^{2} \\
M_{H^{0}, h^{0}}^{2} & =\frac{1}{2}\left(M_{A^{0}}^{2}+M_{Z}^{2}+\Omega+\Sigma \pm \sqrt{R}\right)
\end{aligned}
$$



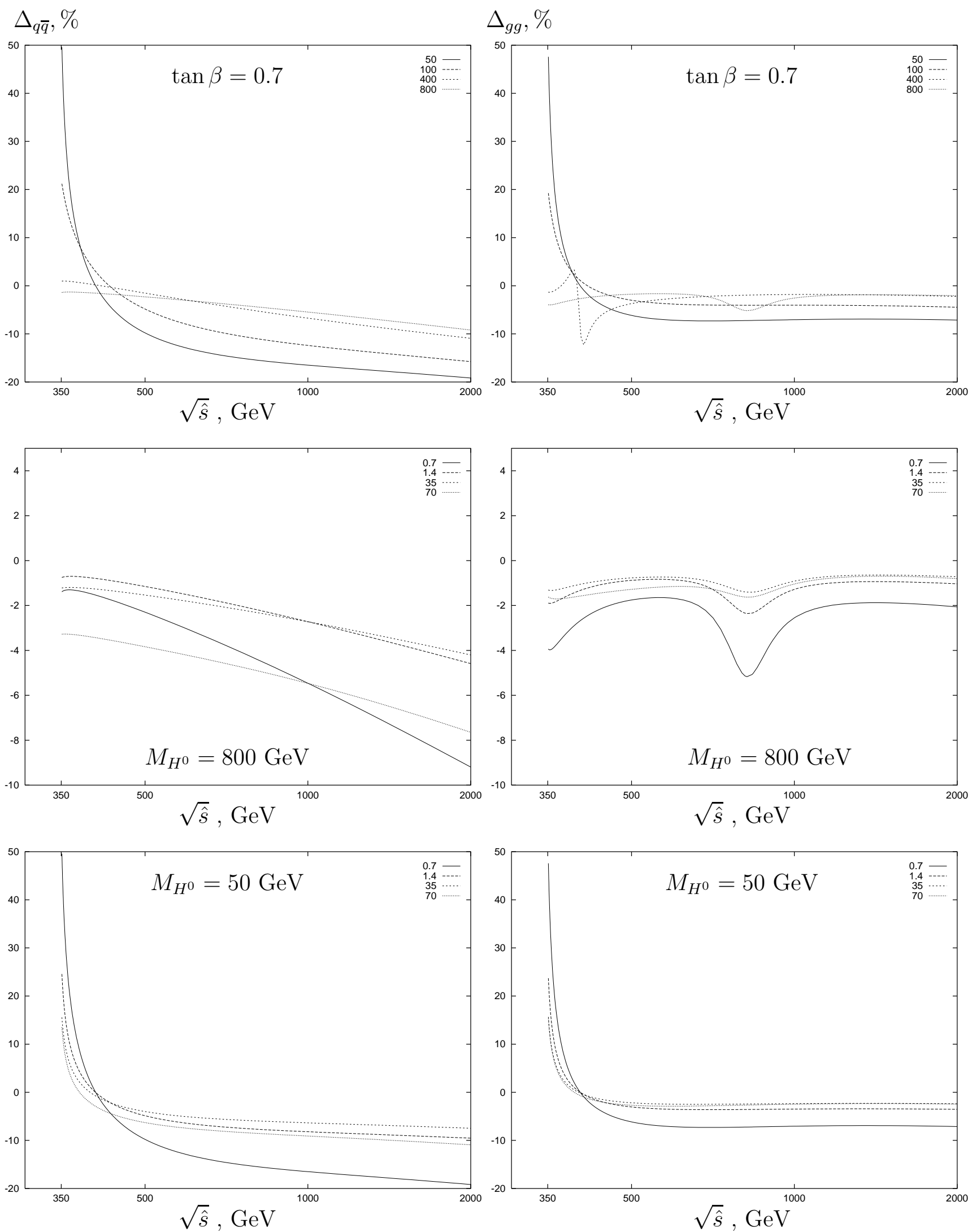

FIG. 3. The variation of the relative corrections $\Delta_{q \bar{q}}$ and $\Delta_{g g}$ with $M_{H^{0}}$ and $\tan \beta$ within the G2HDM (with $M_{h^{0}}=45 \mathrm{GeV}, M_{A^{0}}=50 \mathrm{GeV}, M_{H^{ \pm}}=50 \mathrm{GeV}$ and $\alpha=\pi / 2$ ). 


$$
\tan \alpha_{e f f}=\frac{\Lambda-\sin \beta \cos \beta\left(M_{A^{0}}^{2}+M_{Z}^{2}\right)}{M_{Z}^{2} \cos ^{2} \beta+M_{A^{0}}^{2} \sin ^{2} \beta+\Sigma-M_{h^{0}}^{2}}
$$

with

$$
\begin{aligned}
R & =\left(M_{A^{0}}^{2}+M_{Z}^{2}\right)^{2}-4 M_{A^{0}}^{2} M_{Z}^{2} \cos ^{2} 2 \beta+(\Omega-\Sigma)\left(\Omega-\Sigma+2\left(M_{A^{0}}^{2}-M_{Z}^{2}\right) \cos 2 \beta\right) \\
& +4 \Lambda\left(\Lambda-\left(M_{A^{0}}^{2}+M_{Z}^{2}\right) \sin 2 \beta\right) .
\end{aligned}
$$

In the approximation where only the dominating $m_{t}^{4}$-terms have been kept, the one-loop corrections parametrized in terms of $\Lambda, \Sigma$ and $\Omega$ read as follows (at tree-level: $\Lambda=\Sigma=\Omega=$ $0)$ :

$$
\begin{aligned}
\Omega & =v\left[\log \left(\frac{m_{\tilde{t}_{1}} m_{\tilde{t}_{2}}}{m_{t}^{2}}\right)+A_{\tilde{t}} S\left(2 k+A_{\tilde{t}} S g\right)\right] \\
\Sigma & =v g(\mu S)^{2} \\
\Lambda & =-v \mu S 2\left(k+A_{\tilde{t}} S g\right)
\end{aligned}
$$

with

$$
\begin{aligned}
v & =\frac{3 G_{F}}{\sqrt{2} \pi^{2}} \frac{m_{t}^{4}}{\sin ^{2} \beta} \\
k & =\frac{1}{m_{\tilde{t}_{1}}^{2}-m_{\tilde{t}_{2}}^{2}} \log \left(\frac{m_{\tilde{t}_{1}}}{m_{\tilde{t}_{2}}}\right) \\
g & =\frac{1-k\left(m_{\tilde{t}_{1}}^{2}+m_{\tilde{t}_{2}}^{2}\right)}{\left(m_{\tilde{t}_{1}}^{2}-m_{\tilde{t}_{2}}^{2}\right)^{2}} \\
S & =A_{\tilde{t}}-\frac{\mu}{\tan \beta} .
\end{aligned}
$$

Note, that our $\mu$-sign convention is different from the one used in [27]. We neglect the impact of radiative corrections on the charged Higgs-boson mass $M_{H^{ \pm}}$since they do not exceed 10 $\mathrm{GeV}$ in a wide range of the parameter space [28] and as pointed out earlier the relative corrections do not significantly depend on the value of $M_{H^{ \pm}}$. The Higgs-mixing parameter $\mu$ of the superpotential, the soft supersymmetry-breaking squark masses $M_{\tilde{Q}}, M_{\tilde{U}}$ and the trilinear soft supersymmetry-breaking parameter $A_{\tilde{t}}$ are treated as free parameters. They only enter the supersymmetric Higgs-sector via radiative corrections. In Eq. (3) we took into account that the superpartners to the left- and right-handed top quarks, $\tilde{t}_{L}$ and $\tilde{t}_{R}$, are not necessarily mass eigenstates since the mass matrix $\mathcal{M}$ is of non-diagonal form

$$
\left(\begin{array}{ll}
\tilde{t}_{L} & \tilde{t}_{R}
\end{array}\right) \mathcal{M}\left(\begin{array}{c}
\tilde{t}_{L} \\
\tilde{t}_{R}
\end{array}\right)
$$

with

$$
\mathcal{M}=\left(\begin{array}{cc}
M_{\tilde{Q}}^{2}+m_{t}^{2}-\cos 2 \beta\left(M_{Z}^{2}-4 M_{W}^{2}\right) / 6 & m_{t}\left(A_{\tilde{t}}-\mu / \tan \beta\right) \\
m_{t}\left(A_{\tilde{t}}-\mu / \tan \beta\right) & M_{\tilde{U}}^{2}+m_{t}^{2}+2 \cos 2 \beta\left(M_{Z}^{2}-M_{W}^{2}\right) / 3
\end{array}\right) .
$$


Thus, $\tilde{t}_{L}, \tilde{t}_{R}$ can mix so that the physical mass eigenstates $\tilde{t}_{1}, \tilde{t}_{2}$ are model-dependent linear combinations of these states. The latter are obtained by diagonalizing the mass matrix by performing the transformation

$$
\begin{aligned}
& \tilde{t}_{1}=\cos \Phi_{\tilde{t}} \tilde{t}_{L}+\sin \Phi_{\tilde{t}} \tilde{t}_{R} \\
& \tilde{t}_{2}=-\sin \Phi_{\tilde{t}} \tilde{t}_{L}+\cos \Phi_{\tilde{t}} \tilde{t}_{R} .
\end{aligned}
$$

Since the off-diagonal elements of the sfermion mass matrices are proportional to the fermion masses we only consider $L, R$-mixing in the stop quark sector.

We choose the light stop quark mass $m_{\tilde{t}_{2}}$ and the mixing angle $\Phi_{\tilde{t}}$ to be input parameters, so that the remaining parameters of the stop quark sector are determined by equations which relate the elements of $\mathcal{M}$

$$
\mathcal{M}=\left(\begin{array}{ll}
a & b \\
b & d
\end{array}\right)
$$

with its eigenvalues and the mixing angle $\Phi_{\tilde{t}}$

$$
\begin{aligned}
m_{\tilde{t}_{1}, \tilde{t}_{2}}^{2} & =\frac{a+d}{2} \pm \sqrt{\frac{(a-d)^{2}}{4}+b^{2}} \\
m_{\tilde{t}_{1}}^{2} & =\cos ^{2} \Phi_{\tilde{t}} a+\sin ^{2} \Phi_{\tilde{t}} d+2 \cos \Phi_{\tilde{t}} \sin \Phi_{\tilde{t}} b \\
m_{\tilde{t}_{2}}^{2} & =\sin ^{2} \Phi_{\tilde{t}} a+\cos ^{2} \Phi_{\tilde{t}} d-2 \cos \Phi_{\tilde{t}} \sin \Phi_{\tilde{t}} b .
\end{aligned}
$$

The soft-supersymmetry-breaking parameter $M_{\tilde{Q}}$ (and thus $a$ ) is already fixed by the mass of the left-handed sbottom quark and $\tan \beta$ (no $\tilde{b}_{L}-\tilde{b}_{R}$-mixing and $m_{\tilde{b}_{L}}=m_{\tilde{b}_{R}}$ )

$$
M_{\tilde{Q}}^{2}=m_{\tilde{b}_{L}}^{2}-m_{b}^{2}+\frac{\cos 2 \beta}{6}\left(M_{Z}^{2}+2 M_{W}^{2}\right) .
$$

Consequently, with the choice of $\Phi_{\tilde{t}}, m_{\tilde{t}_{2}}, m_{\tilde{b}_{L}}$ and $\tan \beta$ the off-diagonal element $b$ and the heavy stop quark mass $m_{\tilde{t}_{1}}$ are fixed. To summarize, the predictions of the supersymmetric 2-Higgs-Doublet Model depend on two parameters of the Higgs-sector which are conventionally chosen to be $M_{A^{0}}$ and $\tan \beta$ and when considering radiative corrections to the supersymmetric mass relations also on $m_{\tilde{b}_{L}}, \mu, \Phi_{\tilde{t}}$ and $m_{\tilde{t}_{2}}$. In the special case of no $L, R$-mixing $\left(\Phi_{\tilde{t}}=0, S=0\right)$ there is no $\mu$-dependence and $\Sigma=\Lambda=0$.

\section{The SUSY EW-like one-loop corrections}

In the following we present the $\mathcal{O}(\alpha)$ contribution of the gaugino-Higgsino-sector within the MSSM to the $q \bar{q}$ annihilation and gluon fusion subprocesses. At one-loop level the $g t \bar{t}$-vertex is modified due to the exchange of two charginos $\tilde{\chi}_{i=1,2}^{ \pm}$and four neutralinos $\tilde{\chi}_{i=1 \cdots 4}^{0}$. They are linear combinations of the supersymmetric partners of the electroweak gauge bosons and Higgs-bosons, the gauginos and Higgsinos, and are obtained by diagonalizing the corresponding mixing matrices $X, Y$ (mass eigenstates $\tilde{\chi}^{0}, \tilde{\chi}^{ \pm}$in four-component notation) [30]: 

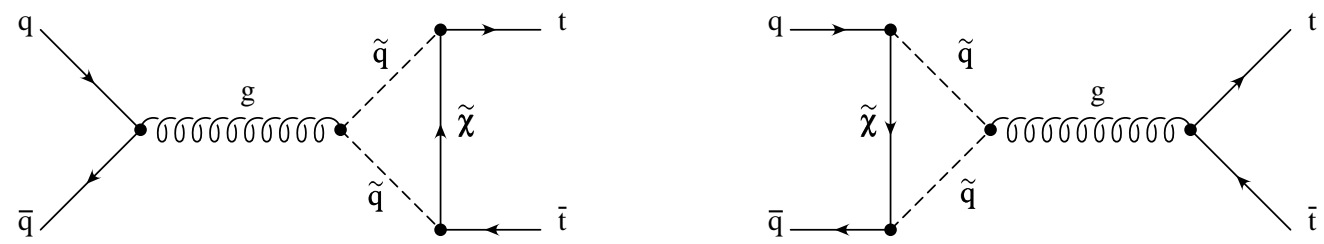

FIG. 4. The Feynman-diagrams for the SUSY EW-like contributions to the $q \bar{q}$ annihilation subprocess.

$$
\mathcal{L}_{m}=-\frac{1}{2} \sum_{i} \overline{\tilde{\chi}}_{i}^{0}\left(N^{*} Y N^{-1}\right)_{i i} \tilde{\chi}_{i}^{0}
$$

with

$$
Y=\left(\begin{array}{cccc}
M_{1} & 0 & -M_{Z} s_{W} \cos \beta & M_{Z} s_{W} \sin \beta \\
0 & M_{2} & M_{Z} c_{W} \cos \beta & -M_{Z} c_{W} \sin \beta \\
-M_{Z} s_{W} \cos \beta & M_{Z} c_{W} \cos \beta & 0 & -\mu \\
M_{Z} s_{W} \sin \beta & -M_{Z} c_{W} \sin \beta & -\mu & 0
\end{array}\right)
$$

and the mass term for the charginos

$$
\mathcal{L}_{m}=-\sum_{i} \overline{\tilde{\chi}}_{i}^{+}\left(U^{*} X V^{-1}\right)_{i i} \tilde{\chi}_{i}^{+}
$$

with

$$
X=\left(\begin{array}{cc}
M_{2} & M_{W} \sqrt{2} \sin \beta \\
M_{W} \sqrt{2} \cos \beta & \mu
\end{array}\right) .
$$

As a result the mass eigenstates $\tilde{\chi}_{i}^{0}, \tilde{\chi}_{i}^{ \pm}$are model-dependent with masses $M_{\tilde{\chi}_{i}^{0}}, M_{\tilde{\chi}_{i}^{ \pm}}$depending on the SUSY parameters $\mu, M_{1}, M_{2}$ and on $\tan \beta$. In order to reduce the number of independent parameters we assume the $S U(2) \times U(1)$ theory being embedded in a grand unified theory so that the following relation becomes valid [30]:

$$
M_{1}=\frac{5 s_{W}}{3 c_{W}} M_{2}
$$

$M_{\tilde{\chi}_{i}^{ \pm}}$and $M_{\tilde{\chi}_{i}^{0}}$ are consequently fixed by the choice of $\mu, M_{2}$ and $\tan \beta$. The diagonalizing matrices for the charginos $U, V$ are real and can be easily chosen so that only positive mass eigenvalues $M_{\tilde{\chi}_{i}^{ \pm}}$occur. Explicit expressions can be found in [30]. Different from the notation of [29,30] we chose the diagonalizing matrix $N$ to be real and allow negative mass eigenvalues $M_{\tilde{\chi}_{i}^{0}}$. The according Feynman-rules involving a neutralino can be retrieved from [29, 30] by performing the transformation described in Appendix A.3 of [29] (here the real matrix $Z$ used in [29] is denoted by $N$ ).

The Feynman-diagrams describing the SUSY EW-like one-loop corrections to the $q \bar{q}$ annihilation and the gluon fusion subprocesses are shown in Fig. 4 and Fig. 5, respectively. The Feynman-rules for the arising triple and quartic squark-gluon interactions are given in Fig. 6 [30]. By introducing the coupling parameters $g_{s, p}^{j}$ with $j=L, R$ (without 

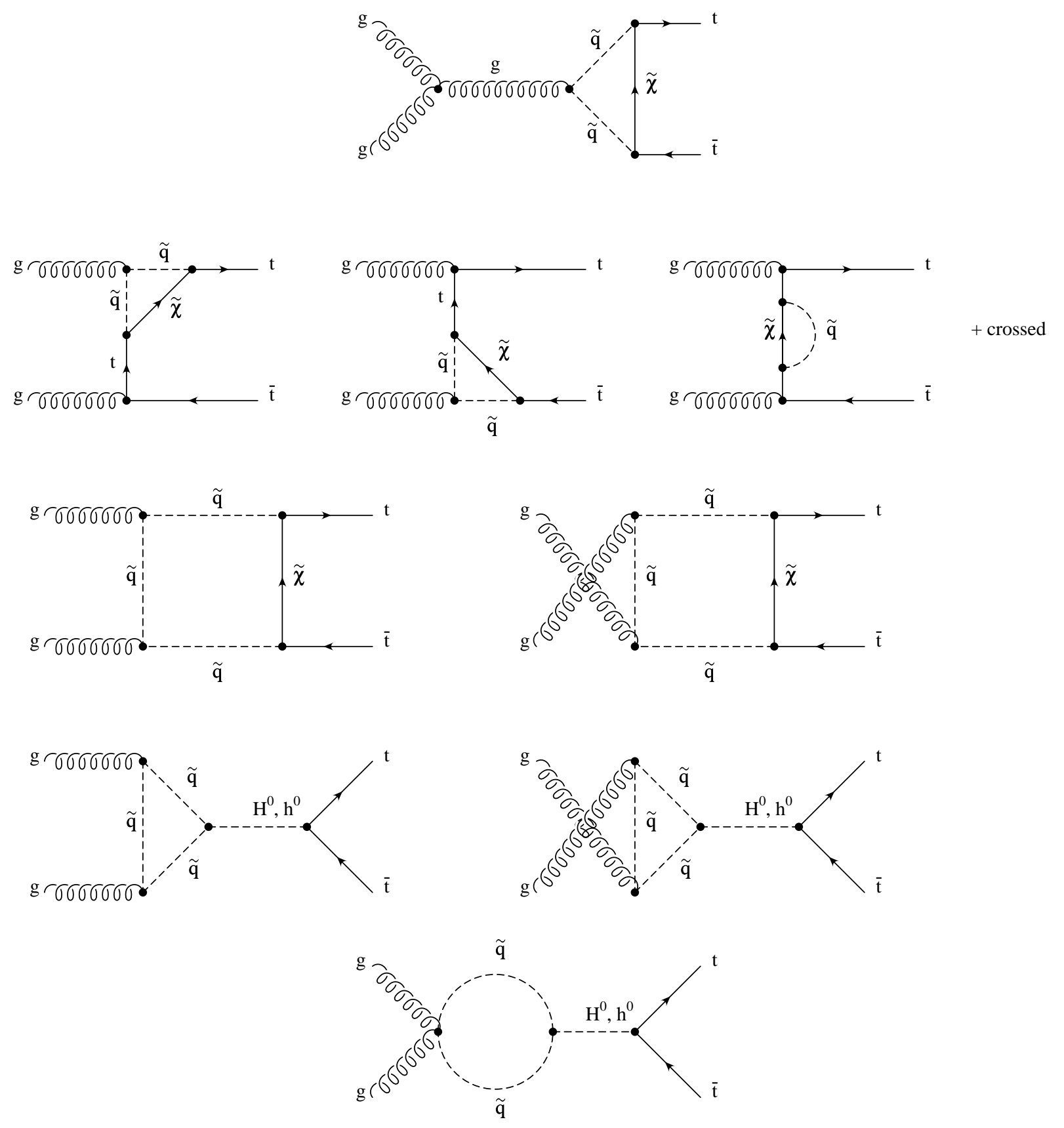

FIG. 5. The Feynman-diagrams for the SUSY EW-like contributions to the gluon fusion subprocess. 

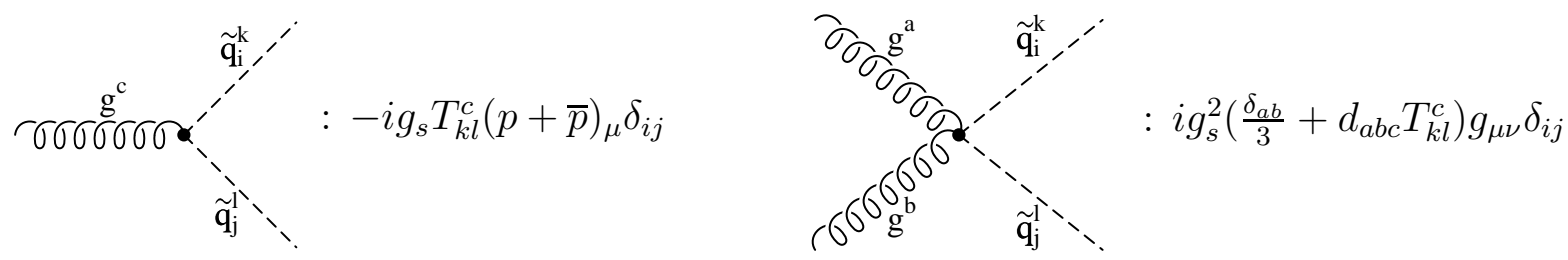

FIG. 6. The Feynman-rules for the triple and quartic squark-gluon interactions. $a, b, c=1 \cdots 8$ and $k, l=1 \cdots 3$ are color indices and $i, j=L, R . g_{s}^{2}=4 \pi \alpha_{s}$ denotes the strong coupling parameter and $T^{c}=\lambda^{c} / 2$ with the Gell-Mann-matrices $\lambda^{c}$ satisfying the $S U(3)$ anticommutation relation $2 d_{a b c} \lambda^{c}=\left\{\lambda_{a}, \lambda_{b}\right\}-4 / 3 \delta_{a b}$.
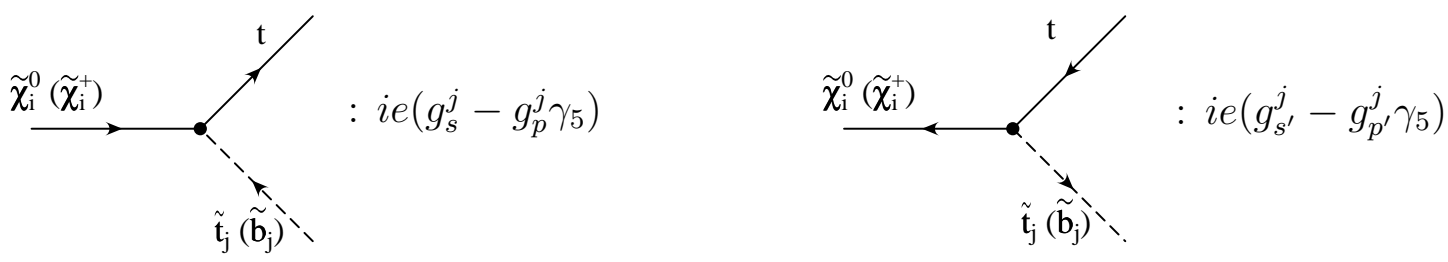

FIG. 7. The $\chi_{i}^{0, \pm}-\tilde{t}_{j}\left(\tilde{b}_{j}\right)-t$ - vertex written in generalized form. The coupling parameters $g_{s, p}^{j}$ are explicitly given in Tab. II.

mixing) and $j=1,2$ (with mixing) the neutralino(chargino)-stop(sbottom)-top-vertex can again be written in a generalized form as shown in Fig. 7 . When considering $\tilde{t}_{L}-\tilde{t}_{R}$-mixing the interaction eigenstates are replaced by the mass eigenstates by using the transformation of Eq. (9) in the interaction Lagrangian, which has the following impact on the stop quark couplings of Tab. 匹:

$$
\begin{aligned}
& g_{s}^{1,2}=\cos \Phi_{\tilde{t}} g_{s}^{L, R} \pm \sin \Phi_{\tilde{t}} g_{s}^{R, L} \\
& g_{p}^{1,2}=\cos \Phi_{\tilde{t}} g_{p}^{L, R} \pm \sin \Phi_{\tilde{t}} g_{p}^{R, L}
\end{aligned}
$$

With these Feynman-rules and after taking into account the counter terms of the on-shell renormalization procedure the SUSY EW-like one-loop corrections to the $q \bar{q}$ annihilation subprocess can be described by means of finite form factors $F_{V}, F_{M}$ (introduced in [11]) which modify the $g t \bar{t}$-vertex

$$
F_{V, M}(\hat{s})=\sum_{j=1}^{2}\left(\sum_{i=1}^{4} S_{V, M}\left(m_{\tilde{t}_{j}}, M_{\tilde{\chi}_{i}^{0}}\right)+\sum_{i=1}^{2} S_{V, M}\left(m_{\tilde{b}_{j}}, M_{\tilde{\chi}_{i}^{ \pm}}\right)\right)
$$

with

$$
\begin{aligned}
S_{V}\left(m_{1}, m_{2}\right) & =\lambda_{j}^{+} 2 C_{2}^{0}\left(\hat{s}, m_{1}, m_{1}, m_{2}\right)+\delta Z_{V} \\
S_{M}\left(m_{1}, m_{2}\right) & =\lambda_{j}^{+}\left(-4 m_{t}^{2}\right)\left(C_{1}^{-}+2 C_{2}^{-}\right)\left(\hat{s}, m_{1}, m_{1}, m_{2}\right) \\
& +\lambda_{j}^{-} 2 m_{t} m_{2}\left(2 C_{1}^{-}+C_{0}\right)\left(\hat{s}, m_{1}, m_{1}, m_{2}\right),
\end{aligned}
$$

where we used the abbreviation $\lambda_{j}^{ \pm}=\left(g_{s}^{j}\right)^{2} \pm\left(g_{p}^{j}\right)^{2}$. The renormalization constant $\delta Z_{V}$ is 
TABLE II. The scalar and pseudo-scalar couplings $g_{s}^{L, R}$ and $g_{p}^{L, R}$ describing the neutralino(chargino)-left (right)handed stop(sbottom)-top-vertex. For convenience a matrix $N^{\prime}$ with $N_{i 1, i 2}^{\prime}=c_{W} N_{i 1, i 2} \pm s_{W} N_{i 2, i 1}$ has been introduced.

\begin{tabular}{|lcc|}
\hline \hline \multicolumn{3}{|c|}{$g_{s, p}^{j}=a \pm b ; g_{s^{\prime}}^{j}=g_{s}^{j} ; g_{p^{\prime}}^{j}=-g_{p}^{j}$} \\
\hline \hline$a$ & $b$ \\
\hline$\tilde{\chi}_{i}^{0} \tilde{t}_{L} t$ & $-N_{i 4} m_{t} /\left(2 \sqrt{2} s_{W} M_{W} \sin \beta\right)$ & $-\frac{1}{\sqrt{2}}\left(\frac{2}{3} N_{i 1}^{\prime}+\frac{1-\frac{4}{3} s_{W}^{2}}{2 c_{W} s_{W}} N_{i 2}^{\prime}\right)$ \\
\hline$\tilde{\chi}_{i}^{0} \tilde{t}_{R} t$ & $\frac{1}{\sqrt{2}} \frac{2}{3}\left(N_{i 1}^{\prime}-\frac{s_{W}}{c_{W}} N_{i 2}^{\prime}\right)$ & $-N_{i 4} m_{t} /\left(2 \sqrt{2} s_{W} M_{W} \sin \beta\right)$ \\
\hline$\tilde{\chi}_{i}^{+} \tilde{b}_{L} t$ & $V_{i 2} m_{t} /\left(2 \sqrt{2} s_{W} M_{W} \sin \beta\right)$ & $-\frac{1}{2 s_{W}} U_{i 1}$ \\
\hline$\tilde{\chi}_{i}^{+} \tilde{b}_{R} t$ & 0 & $U_{i 2} m_{b} /\left(2 \sqrt{2} s_{W} M_{W} \cos \beta\right)$ \\
\hline \hline
\end{tabular}

determined by the top quark self-energy $\Sigma_{V, S}$

$$
\delta Z_{V}=-\Sigma_{V}\left(p^{2}=m_{t}^{2}\right)-2 m_{t}^{2} \frac{\partial}{\partial p^{2}}\left(\Sigma_{V}+\Sigma_{S}\right)_{p^{2}=m_{t}^{2}}
$$

with

$$
\begin{aligned}
& \Sigma_{V}\left(p^{2}\right)=-\lambda_{j}^{+} B_{1}\left(p^{2}, m_{2}, m_{1}\right) \\
& \Sigma_{S}\left(p^{2}\right)=\lambda_{j}^{-} \frac{m_{2}}{m_{t}} B_{0}\left(p^{2}, m_{2}, m_{1}\right) .
\end{aligned}
$$

The Feynman-diagrams of Fig. 5 represent the SUSY EW-like $\mathcal{O}(\alpha)$ contribution to the gluon fusion subprocess consisting of the modification of the $g t \bar{t}$-vertex in the $s$ - and $t$-production channel described by the form factors $F_{V}, F_{M}$ and $\rho_{i}^{V}$, respectively, the selfenergy insertion to the off-shell top quark $\left(\rho_{i}^{\Sigma}\right)$, the UV-finite box diagrams $\left(\rho_{i}^{\square}\right)$ and the UV-finite $s$-channel Higgs-exchange $\left(\rho_{i}^{\triangle}\right)$. These contributions together with the counter terms modify the gluon fusion form factors in [11] as follows:

$$
\rho_{i}^{(V, \Sigma, \square),(t, u)}=\sum_{j=1}^{2}\left(\sum_{i=1}^{4} T_{i}^{(V, \Sigma, \square),(t, u)}\left(m_{\tilde{t}_{j}}, M_{\tilde{\chi}_{i}^{0}}\right)+\sum_{i=1}^{2} T_{i}^{(V, \Sigma, \square),(t, u)}\left(m_{\tilde{b}_{j}}, M_{\tilde{\chi}_{i}^{ \pm}}\right)\right)
$$

with

$\underline{\text { Vertex corrections: }}$

$$
\begin{aligned}
T_{1}^{V, t}\left(m_{1}, m_{2}\right) & =4 \lambda_{j}^{+} C_{2}^{0}\left(\hat{t}, m_{1}, m_{1}, m_{2}\right)+2 \delta Z_{V} \\
T_{4}^{V, t}\left(m_{1}, m_{2}\right) & =2 \lambda_{j}^{+}\left(\hat{t}-m_{t}^{2}\right)\left(C_{2}^{2}-C_{2}^{12}+C_{1}^{2}\right)\left(\hat{t}, m_{1}, m_{1}, m_{2}\right) \\
T_{11}^{V, t}\left(m_{1}, m_{2}\right) & =-T_{1}^{V, t}\left(m_{1}, m_{2}\right) \\
T_{14}^{V, t}\left(m_{1}, m_{2}\right) & =2 \lambda_{j}^{+}\left(-C_{2}^{1}-C_{2}^{2}+2 C_{2}^{12}-C_{1}^{1}-C_{1}^{2}\right)\left(\hat{t}, m_{1}, m_{1}, m_{2}\right) \\
& +2 \lambda_{j}^{-} \frac{m_{2}}{m_{t}}\left(C_{1}^{1}+C_{1}^{2}+C_{0}\right)\left(\hat{t}, m_{1}, m_{1}, m_{2}\right) \\
T_{16}^{V, t}\left(m_{1}, m_{2}\right) & =-4 T_{14}^{V, t}\left(m_{1}, m_{2}\right)
\end{aligned}
$$


$\underline{\text { Top quark self-energy insertion: }}$

$$
\begin{aligned}
T_{1}^{\Sigma, t}\left(m_{1}, m_{2}\right) & =-\left(\hat{t}+m_{t}^{2}\right)\left(\Sigma_{V}\left(\hat{t}, m_{2}, m_{1}\right)+\delta Z_{V}\right) \\
& -2 m_{t}^{2}\left(\Sigma_{S}(\hat{t})-\delta Z_{V}-\Sigma_{S}\left(m_{t}^{2}\right)-\Sigma_{V}\left(m_{t}^{2}\right)\right) \\
T_{11}^{\Sigma, t}\left(m_{1}, m_{2}\right) & =2 \hat{t}\left(\Sigma_{V}\left(\hat{t}, m_{2}, m_{1}\right)+\delta Z_{V}\right) \\
& +\left(\hat{t}+m_{t}^{2}\right)\left(\Sigma_{S}(\hat{t})-\delta Z_{V}-\Sigma_{S}\left(m_{t}^{2}\right)-\Sigma_{V}\left(m_{t}^{2}\right)\right)
\end{aligned}
$$

Box contribution:

$$
\begin{aligned}
T_{2}^{\square, t}\left(m_{1}, m_{2}\right) & =2 \lambda_{j}^{+} D_{3}^{02}\left(\hat{t}, m_{1}, m_{1}, m_{1}, m_{2}\right) \\
T_{4}^{\square, t}\left(m_{1}, m_{2}\right) & =4 \lambda_{j}^{+}\left(D_{2}^{0}+2 D_{3}^{01}+D_{3}^{02}\right)\left(\hat{t}, m_{1}, m_{1}, m_{1}, m_{2}\right) \\
T_{6}^{\square, t}\left(m_{1}, m_{2}\right) & =-2 \lambda_{j}^{+}\left(D_{1}^{2}+D_{3}^{2}+2\left(2 D_{2}^{12}+D_{2}^{2}+D_{3}^{12}+D_{3}^{123}-2 D_{3}^{21}\right)\right)\left(\hat{t}, m_{1}, m_{1}, m_{1}, m_{2}\right) \\
T_{12}^{\square, t}\left(m_{1}, m_{2}\right) & =-4 \lambda_{j}^{+}\left(2 D_{3}^{01}+D_{3}^{02}\right)\left(\hat{t}, m_{1}, m_{1}, m_{1}, m_{2}\right)+4 \lambda_{j}^{-} \frac{m_{2}}{m_{t}} D_{2}^{0}\left(\hat{t}, m_{1}, m_{1}, m_{1}, m_{2}\right) \\
T_{16}^{\square, t}\left(m_{1}, m_{2}\right) & =4 \lambda_{j}^{+}\left(D_{1}^{2}+D_{3}^{2}+2\left(D_{1}^{1}+2 D_{2}^{1}+4 D_{2}^{12}+2 D_{2}^{13}+D_{2}^{2}+D_{3}^{1}\right.\right. \\
& \left.\left.+3 D_{3}^{12}+3 D_{3}^{123}+3 D_{3}^{13}+3 D_{3}^{21}\right)\right)\left(\hat{t}, m_{1}, m_{1}, m_{1}, m_{2}\right) \\
& -4 \lambda_{j}^{-} \frac{m_{2}}{m_{t}}\left(D_{0}+D_{2}^{2}+2\left(2 D_{1}^{1}+D_{1}^{2}+D_{2}^{1}+2 D_{2}^{12}+D_{2}^{13}\right)\right)\left(\hat{t}, m_{1}, m_{1}, m_{1}, m_{2}\right) .
\end{aligned}
$$

$\delta Z_{V}$ and $\Sigma_{V, S}\left(p^{2}\right)$ are given by Eq. 22 and Eq. 23, respectively. The $u$-channel contribution $\rho_{i}^{(V, \Sigma, \square), u}$ can be obtained from the $t$-channel form factors by replacing $\hat{t}$ with $\hat{u}$. For the notations concerning the $B, C, D$-functions see [11]. The $s$-channel Higgs-exchange contribution is described by:

$$
\begin{aligned}
\rho_{12}= & \frac{m_{t}}{2 s_{W} M_{W}} \sum_{S=H^{0}, h^{0}} \sum_{j=1,2} \sum_{q} \frac{c_{s} c_{s, j}^{\text {susy }}}{m_{t}}\left(\hat{s}-M_{S}^{2}-i M_{S} \Gamma_{S}\right) \\
& {\left[-2 m_{\tilde{q}_{j}}^{2} C_{0}\left(\hat{s}, m_{\tilde{q}_{j}}, m_{\tilde{q}_{j}}, m_{\tilde{q}_{j}}\right)-1\right] }
\end{aligned}
$$

with 13

$$
C_{0}\left(\hat{s}, m_{\tilde{q}_{j}}, m_{\tilde{q}_{j}}, m_{\tilde{q}_{j}}\right)=\left\{\begin{array}{c}
\frac{1}{2 \hat{s}}\left(\log \frac{1+\sqrt{1-\tau}}{1-\sqrt{1-\tau}}-i \pi\right)^{2} ; \tau=\frac{4 m_{\tilde{q}_{j}}^{2}}{\hat{s}}<1 \\
-\frac{2}{\hat{s}}\left(\sin ^{-1} \sqrt{\frac{1}{\tau}}\right)^{2} ; \tau \geq 1
\end{array}\right.
$$

where $c_{s}$ is taken from Tab. [ and $c_{s, j}^{\text {susy }}$ from Tab. [II. The tree level decay widths of the light and heavy neutral Higgs-bosons $\Gamma_{h^{0}, H^{0}}$ within the MSSM are given in [13. In the stop quark sector when taking into account $\tilde{t}_{L}-\tilde{t}_{R}$-mixing $c_{s,(L L, R R)}^{\text {susy }}$ is replaced with

$$
c_{s,(1,2)}^{\text {susy }}=\cos ^{2} \Phi_{\tilde{t}} c_{s,(L L, R R)}^{\text {susy }}+\sin ^{2} \Phi_{\tilde{t}} c_{s,(R R, L L)}^{\text {susy }} \pm 2 \sin \Phi_{\tilde{t}} \cos \Phi_{\tilde{t}} c_{s, L R}^{\text {susy }} .
$$

The masses of the supersymmetric partners to the light quarks in the unmixed up-type and down-type quark sector are again fixed by choosing $m_{\tilde{b}_{L}}$ and assuming that $m_{\tilde{q}_{L}}=m_{\tilde{q}_{R}}$. The corresponding light quark masses are considered to be zero. 
TABLE III. Higgs-boson couplings to left and right-handed squarks within the MSSM (with $c_{+}=\cos (\alpha+\beta), s_{+}=\sin (\alpha+\beta)$ and $\left.A_{\tilde{f} \neq \tilde{t}}=\mu / \tan \beta\right)$

\begin{tabular}{|ccc|}
\hline \hline & $c_{s,(L L, R R, L R)}^{\text {susy }}$ \\
\hline \hline$T_{3}^{f}>0, Q_{f}=2 / 3$ & $T_{3}^{f}<0, Q_{f}=-1 / 3$ \\
\hline$H^{0} \tilde{f}_{L} \tilde{f}_{L}$ & $-\frac{M_{Z}}{c_{W} s_{W}}\left(T_{3}^{f}-Q_{f} s_{W}^{2}\right) c_{+}-\frac{m_{f}^{2}}{M_{W} s_{W}} \frac{\sin \alpha}{\sin \beta}$ & $-\frac{M_{Z}}{c_{W} s_{W}}\left(T_{3}^{f}-Q_{f} s_{W}^{2}\right) c_{+}-\frac{m_{f}^{2}}{M_{W} s_{W}} \frac{\cos \alpha}{\cos \beta}$ \\
\hline$H^{0} \tilde{f}_{R} \tilde{f}_{R}$ & $-\frac{M_{Z}}{c_{W}} s_{W} Q_{f} c_{+}-\frac{m_{f}^{2}}{M_{W} s_{W}} \frac{\sin \alpha}{\sin \beta}$ & $-\frac{M_{Z}}{c_{W}} s_{W} Q_{f} c_{+}-\frac{m_{f}^{2}}{M_{W} s_{W}} \frac{\cos \alpha}{\cos \beta}$ \\
\hline$H^{0} \tilde{f}_{L} \tilde{f}_{R}$ & $-\frac{m_{f}}{2 M_{W} s_{W} \sin \beta}\left(-\mu \cos \alpha+A_{\tilde{f}} \sin \alpha\right)$ & $-\frac{m_{f}}{2 M_{W} s_{W} \cos \beta}\left(-\mu \sin \alpha+A_{\tilde{f}} \cos \alpha\right)$ \\
\hline$h^{0} \tilde{f}_{L} \tilde{f}_{L}$ & $\frac{M_{Z}}{c_{W} s_{W}}\left(T_{3}^{f}-Q_{f} s_{W}^{2}\right) s_{+}-\frac{m_{f}^{2}}{M_{W} s_{W}} \frac{\cos \alpha}{\sin \beta}$ & $\frac{M_{Z}}{c_{W} s_{W}}\left(T_{3}^{f}-Q_{f} s_{W}^{2}\right) s_{+}+\frac{m_{f}^{2}}{M_{W} s_{W}} \frac{\sin \alpha}{\cos \beta}$ \\
\hline$h^{0} \tilde{f}_{R} \tilde{f}_{R}$ & $\frac{M_{Z}}{c_{W}} s_{W} Q_{f} s_{+}-\frac{m_{f}^{2}}{M_{W} s_{W}} \frac{\cos \alpha}{\sin \beta}$ & $\frac{M_{Z}}{c_{W}} s_{W} Q_{f} s_{+}-\frac{m_{f}^{2}}{M_{W} s_{W}} \frac{\sin \alpha}{\cos \beta}$ \\
\hline$h^{0} \tilde{f}_{L} \tilde{f}_{R}$ & $-\frac{m_{f}}{2 M_{W} s_{W} \sin \beta}\left(\mu \sin \alpha+A_{\tilde{f}} \cos \alpha\right)$ & $-\frac{m_{f}}{2 M_{W} s_{W} \cos \beta}\left(-\mu \cos \alpha-A_{\tilde{f}} \sin \alpha\right)$ \\
\hline \hline
\end{tabular}

In order to study the effects of the SUSY EW-like contribution and what remains after including the contribution from the supersymmetric Higgs-sector with special emphasis on the $s$-channel Higgs-exchange diagrams we discuss in Fig. 8 and Fig. 9 the SUSY EW-like one-loop corrections and the full $\mathcal{O}(\alpha)$ MSSM contribution, respectively, to the gluon fusion subprocess. Since the SUSY $s$-channel Higgs-exchange contribution is proportional to the quark masses when the squark masses are considered to be degenerate in mass, only the stop quark-loop contributes significantly. The characteristic structure around $\sqrt{\hat{s}}=M_{H^{0}}$ in the Figs. 8,9 originates from the $s$-channel Higgs-exchange diagrams. Since $M_{h^{0}} \ll \sqrt{\hat{s}}$ we only observe the resonance structure due to the exchange of the heavy neutral Higgs-boson $H^{0}$. As can be seen when comparing the SUSY EW-like with the full MSSM contribution the top quark-loop contribution to the $s$-channel Higgs-exchange diagrams dominates. Only when the SUSY Higgs-decay width $\Gamma_{H^{0} \rightarrow \tilde{q} \tilde{q}, \tilde{\chi} \tilde{\chi}}$ is large the resonance structure arising from the top quark-loop contribution is also suppressed as observed in Fig. 8 and Fig. 9 for $\Phi_{\tilde{t}}=\pi / 4$ and $m_{\tilde{t}_{2}}=75 \mathrm{GeV}$, respectively. In the following we chose $\tan \beta=0.7$ and $M_{A^{0}}=450 \mathrm{GeV}$ $\left(\Rightarrow M_{H^{0}}\right)$. Concerning the $s$-channel Higgs-exchange diagrams the dependence on $\tan \beta$ and $M_{H^{0}}$ is essentially not different from what we observed within the G2HDM.

In Fig. 8 we study the dependence on the light stop quark mass $m_{\tilde{t}_{2}}$ and on the mixing angle $\Phi_{\tilde{t}}$. Both parameters affect the resonance structure via the decay width $\Gamma_{H^{0}}$ and also via the Higgs-boson mass $M_{H^{0}}$ when the described radiative corrections to the supersymmetric Higgs-mass relations are taken into account. Moreover, in the resonance region the negative form factor $\rho_{12}^{\triangle}$ of Eq. (28) increases with $m_{\tilde{t}_{2}}$ and thus decreases the otherwise positive SUSY EW-like contribution. When $\Phi_{\tilde{t}} \neq 0$ is chosen the $L, R$-mixing term in the coupling parameter $c_{s,(1,2)}^{\text {susy }}$ contributes and depending on the sign of $\Phi_{\tilde{t}}$ (more accurate: the sign of $\mu \Phi_{\tilde{t}}$ ) either diminishes or enhances the SUSY $s$-channel Higgs-exchange contribution. 
In Fig. 9 the dependence on $\mu$ and $m_{\tilde{b}_{1}}$ is shown. Again the choice of $\mu$ effects the resonance structure by changing $M_{H^{0}}$. Also in Fig. 9 the enhancement due to the threshold effect in the vicinity of $m_{t}=m_{\tilde{t}_{2}}+M_{\tilde{\chi}^{0}}$ can be observed for $m_{\tilde{t}_{2}}=75 \mathrm{GeV}$ and $\mu=-90 \mathrm{GeV}$. The dependence on $\mu$ is much less significant for larger values of $m_{\tilde{t}_{2}}$. Finally, we discuss the effects of varying the sbottom quark mass $m_{\tilde{b}_{1}}$. The dependence of the $s$-channel Higgsexchange contribution on $m_{\tilde{b}_{1}}$ mainly originates from its impact on the value of $M_{H^{0}}$ and the contribution of the sbottom quark to the decay width $\Gamma_{H^{0} \rightarrow \tilde{q} \tilde{q}}$. Although the $s$-channel Higgs-exchange diagrams provide an interesting Higgs-specific structure its significance will only preveal after having performed the convolution of the partonic cross sections with the parton distribution functions.

\section{The $p \bar{p}, p p \rightarrow t \bar{t} X$ cross section to $\mathcal{O}\left(\alpha \alpha_{s}^{2}\right)$}

The observable hadronic cross section is obtained by convoluting the partonic cross sections of Eq. (11) with parton distribution functions

$$
\sigma(S)=\int_{\frac{4 m_{t}^{2}}{S}}^{1} \frac{d \tau}{\tau}\left(\frac{1}{S} \frac{d L_{q \bar{q}}}{d \tau} \hat{s} \hat{\sigma}_{q \bar{q}}\left(\hat{s}, \alpha_{s}(\mu)\right)+\frac{1}{S} \frac{d L_{g g}}{d \tau} \hat{s} \hat{\sigma}_{g g}\left(\hat{s}, \alpha_{s}(\mu)\right)\right)
$$

with $\tau=x_{1} x_{2}=\hat{s} / S$ and the parton luminosities

$$
\frac{d L_{i j}}{d \tau}=\frac{1}{1+\delta_{i j}} \int_{\tau}^{1} \frac{d x_{1}}{x_{1}}\left[f_{i}\left(x_{1}, Q\right) f_{j}\left(\frac{\tau}{x_{1}}, Q\right)+(1 \leftrightarrow 2)\right]
$$

In the numerical evaluation we use the MRSA set of parton distribution functions [31] with the factorization $(Q)$ and renormalization scale $(\mu)$ chosen to be $Q=\mu=m_{t}$.

In order to avoid numerical instabilities and to take into account that jets originating from the produced top quarks at large scattering angles are better distinguishable from the background we impose a cut on the transverse momentum $p_{t}$ and the pseudo rapidity $\eta$ of the top quark in the cm frame: $p_{t}>20,100 \mathrm{GeV}$ (Tevatron, LHC) and $|\eta|<2.5$. Imposing a transverse momentum cut also enhances the relative correction at hadron level

$$
\sigma(S)=\sigma_{B}(S)+\delta \sigma(S)=\sigma_{B}(1+\Delta)
$$

which again is introduced to reveal the numerical impact of the $\mathcal{O}(\alpha)$ contribution on the observable cross sections. Before we start the numerical discussion in detail we describe the general characteristics of the electroweak one-loop corrections: at the parton level apart from a small region close to the threshold $\sqrt{\hat{s}} \approx 2 m_{t}$, where the $\mathcal{O}(\alpha)$ contribution can be extremely large and positive, the EW-like radiative corrections reduce the leading-order cross

sections the more the larger $\sqrt{\hat{s}}$. At the hadron level, as a result of the interplay between the partonic cross sections and the Bjorken- $x$ quark and gluon distributions the relative corrections are predominantly negative, only at the Tevatron where the $q \bar{q}$ annihilation subprocess dominates small positive contributions arise as a remnant of the large corrections arising in the threshold region.

We start the numerical discussion at hadron level with the MSM prediction. In Fig. 10 the effects of the $\mathcal{O}(\alpha)$ Higgs- and electroweak gauge boson contributions are shown separately. 
$\Delta_{g g}, \%$ SUSY EW-like
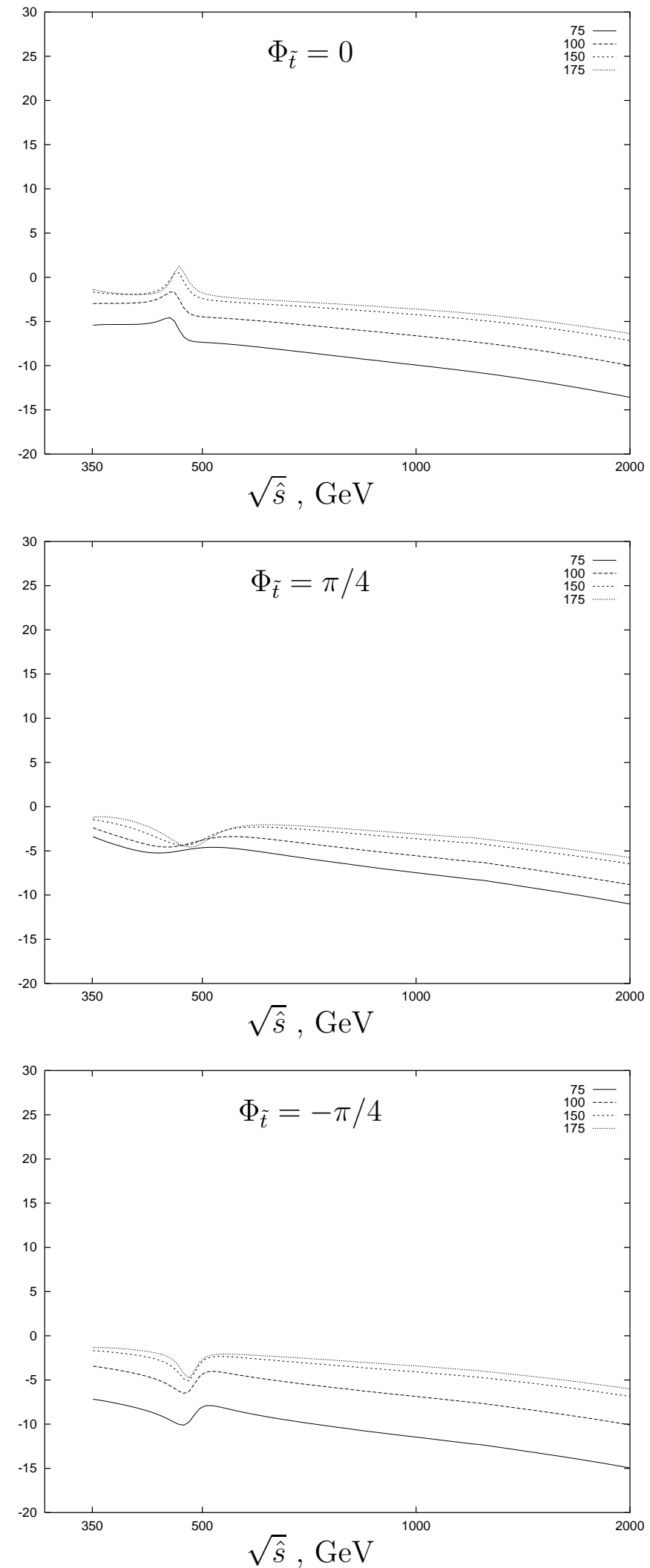

$\Delta_{g g}, \% \mathrm{MSSM}$
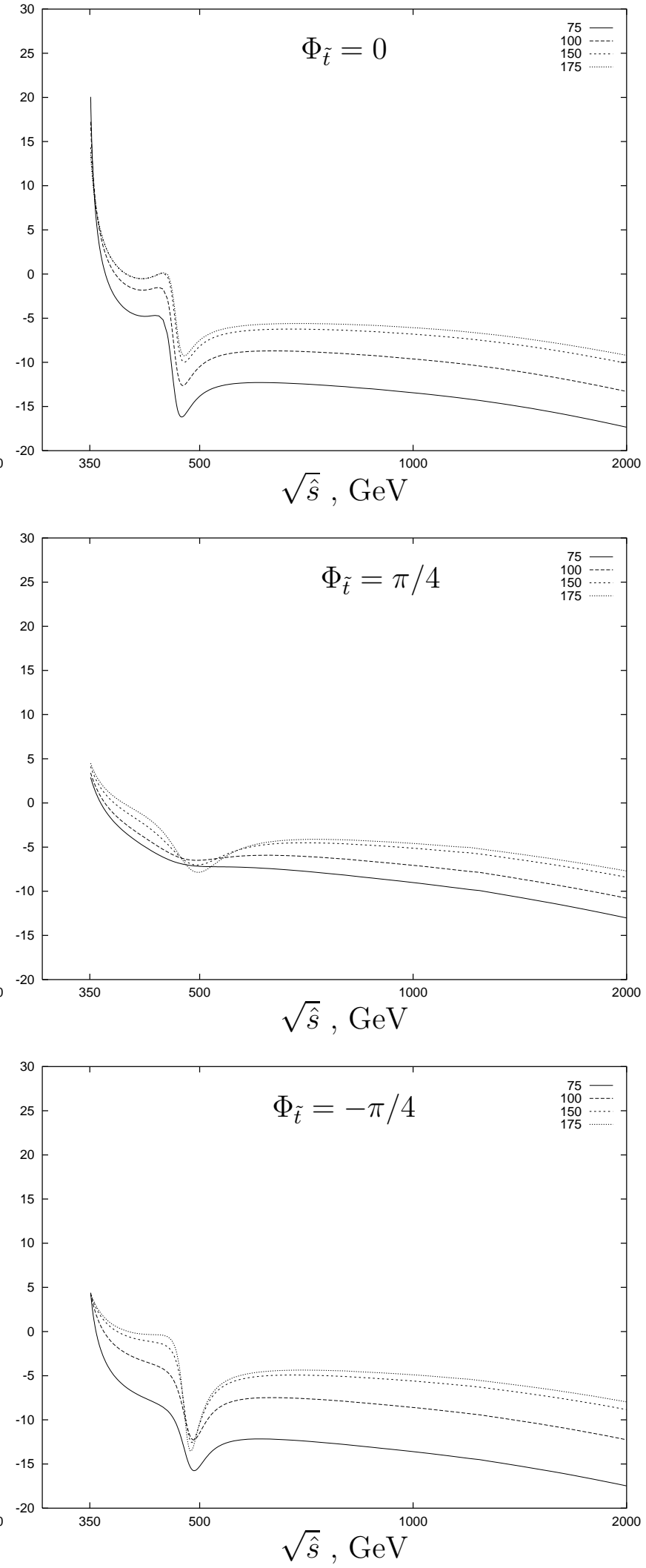

FIG. 8. The variation of the relative correction $\Delta_{g g}$ with $m_{\tilde{t}_{2}}$ for different values of the mixing angle $\Phi_{\tilde{t}}$ (with $M_{A^{0}}=450 \mathrm{GeV}, \tan \beta=0.7, m_{\tilde{b}_{1}}=400 \mathrm{GeV}, \mu=150 \mathrm{GeV}$ and $\left.M_{2}=3|\mu|\right)$. 
$\Delta_{g g}, \%$ SUSY EW-like
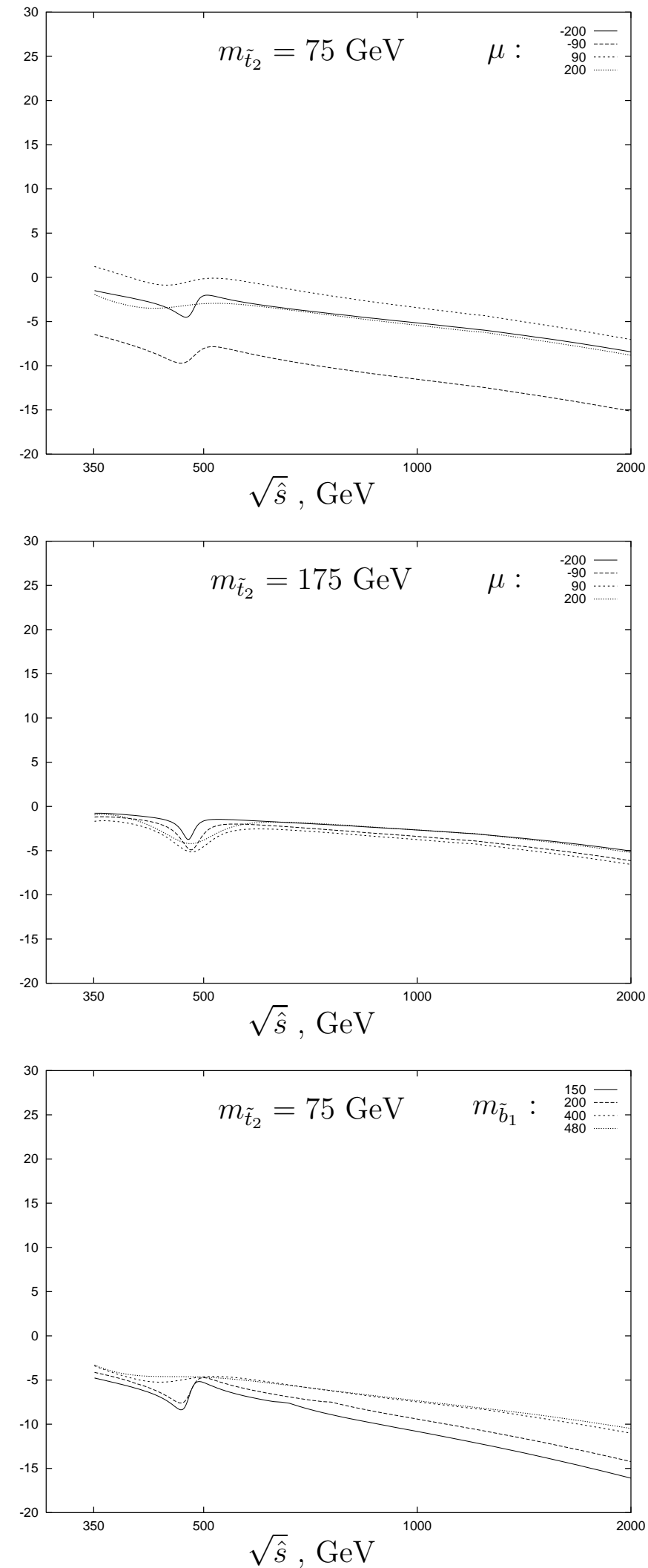

$\Delta_{g g}, \% \mathrm{MSSM}$
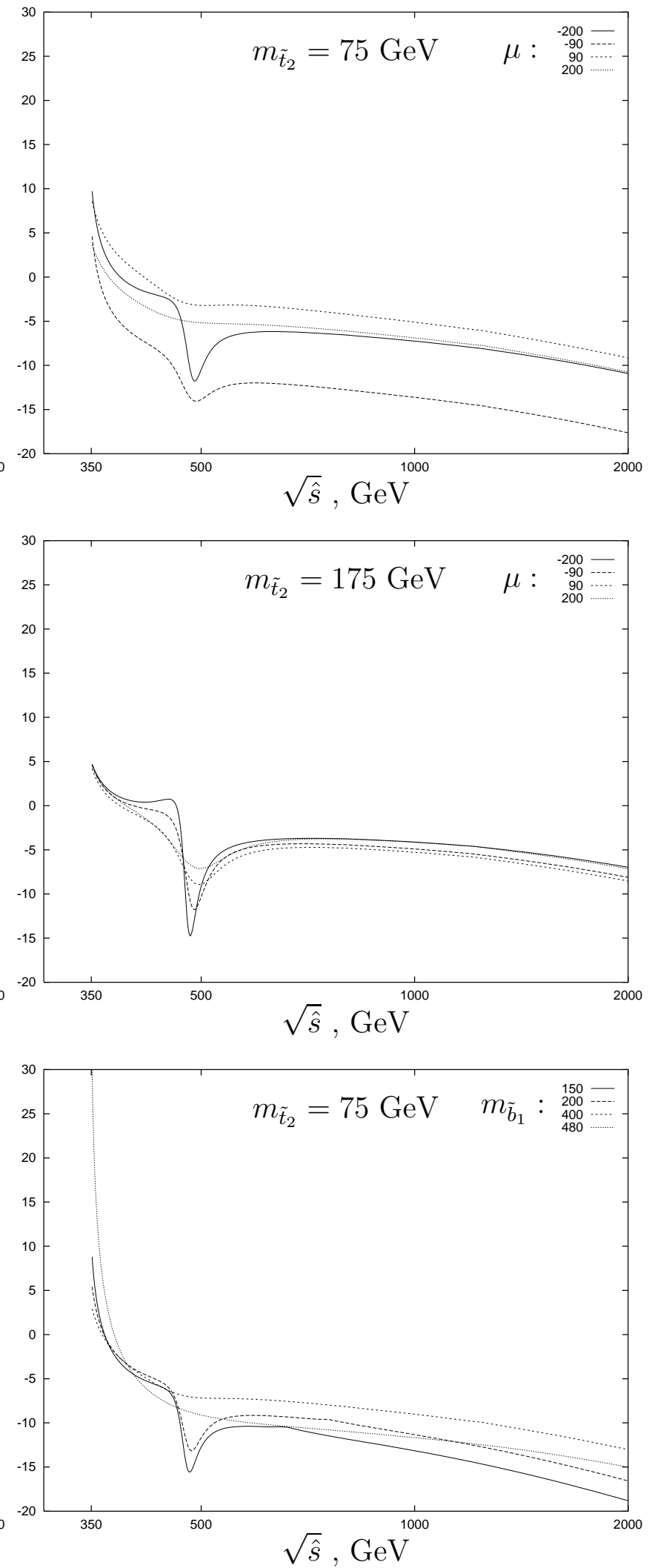

FIG. 9. The variation of the relative correction $\Delta_{g g}$ with $\mu$ for two values of $m_{\tilde{t}_{2}}\left(m_{\tilde{b}_{1}}=400\right.$ $\mathrm{GeV}$ ) and variation with $m_{\tilde{b}_{1}}(\mu=150 \mathrm{GeV})$ (with $M_{2}=3|\mu|, M_{A^{0}}=450 \mathrm{GeV}, \tan \beta=0.7$ and $\left.\Phi_{\tilde{t}}=\pi / 4\right)$. 

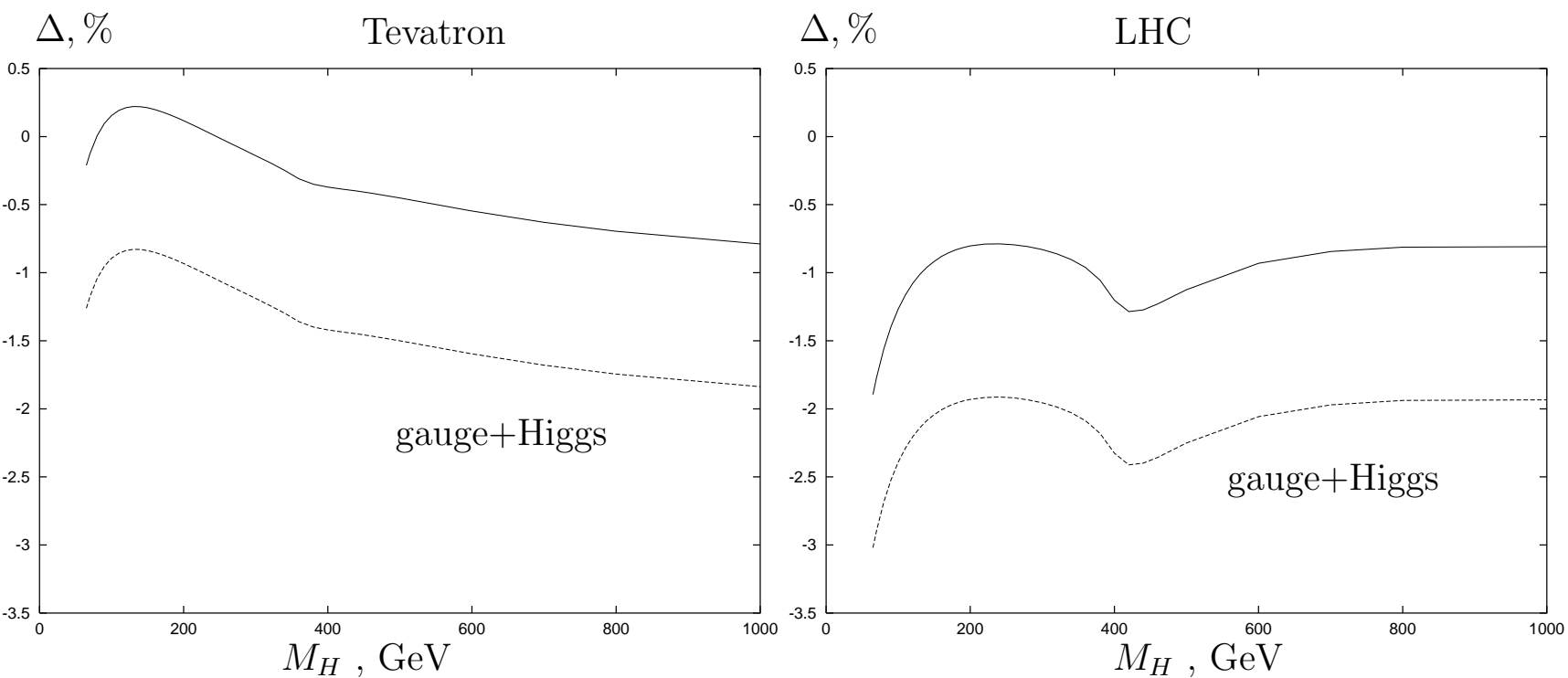

FIG. 10. The variation of the relative correction $\Delta$ with the MSM Higgs-boson mass $M_{H}$ at the Tevatron $\left(S=(2 \mathrm{TeV})^{2}\right)$ and at the $\operatorname{LHC}\left(S=(14 \mathrm{TeV})^{2}\right)$. The $\mathcal{O}(\alpha)$ contribution from the Higgs-sector is shown separately (solid line).

Within the MSM the complete electroweak one-loop corrections diminish the leading order observable cross sections at the Tevatron and the LHC by up to $-2 \%$ and $-3 \%$, respectively. As can be seen in Fig. 10 the consideration of the electroweak gauge boson contribution yield a constant shift of about $-1 \%$ at both colliders. In the following discussion of non-minimal Standard Model implications this effect is not explicitly included, since it can apparently easily be taken into account.

In Fig. 11 we show the G2HDM prediction as a function of $M_{H^{0}}$ for different values of $\tan \beta$. Within the G2HDM due to the enhancement of the Yukawa-couplings by $\tan \beta$ the Born-cross sections can be considerably diminished: at the Tevatron up to $-3.5 \%(\tan \beta=$ 70 and $M_{H^{0}}$ either very small or very high) and at the LHC up to $-7.3 \%(\tan \beta=0.7$ and $H^{0}$ very light). Except for very small values of $M_{H^{0}}$ the contribution only weakly depends on $M_{H^{0}}$. As already discussed at the parton level, the new increase for $\tan \beta=70$ is due to the enhancement of the top-Yukawa-coupling to the charged Higgs-boson. The possibility of a further enhancement of the radiative corrections due to a discontinuity in the derivative of the $B$-functions in the vicinity of the threshold for top quark decay $t \rightarrow b+H^{+}$is illustrated in Fig. 12. There we show the relative corrections as a function of the charged Higgs-boson mass $M_{H^{ \pm}}$for different values of $\tan \beta$.

Within the MSSM the freedom of the G2HDM in choosing that set of parameters which yield the maximum effect is limited by imposing supersymmetric constraints on the 2-HiggsDoublet Model. Thus, the effects of the supersymmetric Higgs-sector are in general less pronounced than the one observed in the G2HDM. This is illustrated in Fig. 13, where the relative corrections obtained within the supersymmetric 2-Higgs-Doublet Model are shown as a function of $M_{A^{0}}$ for different values of $\tan \beta$. In Fig. 14 and Fig. 15 we illustrate the most pronounced effect of the SUSY EW-like contributions: the enhancement of the radiative corrections when $m_{t} \approx m_{\tilde{t}_{2}}+M_{\tilde{\chi}^{0}}$. We show the relative corrections versus $m_{\tilde{t}_{2}}$ for different values of $\tan \beta$ with and without mixing. The absence of the second dip in Fig. 14 illustrates the possibility of a cancellation of the large contributions between the derivative of the $B_{0}$ and 

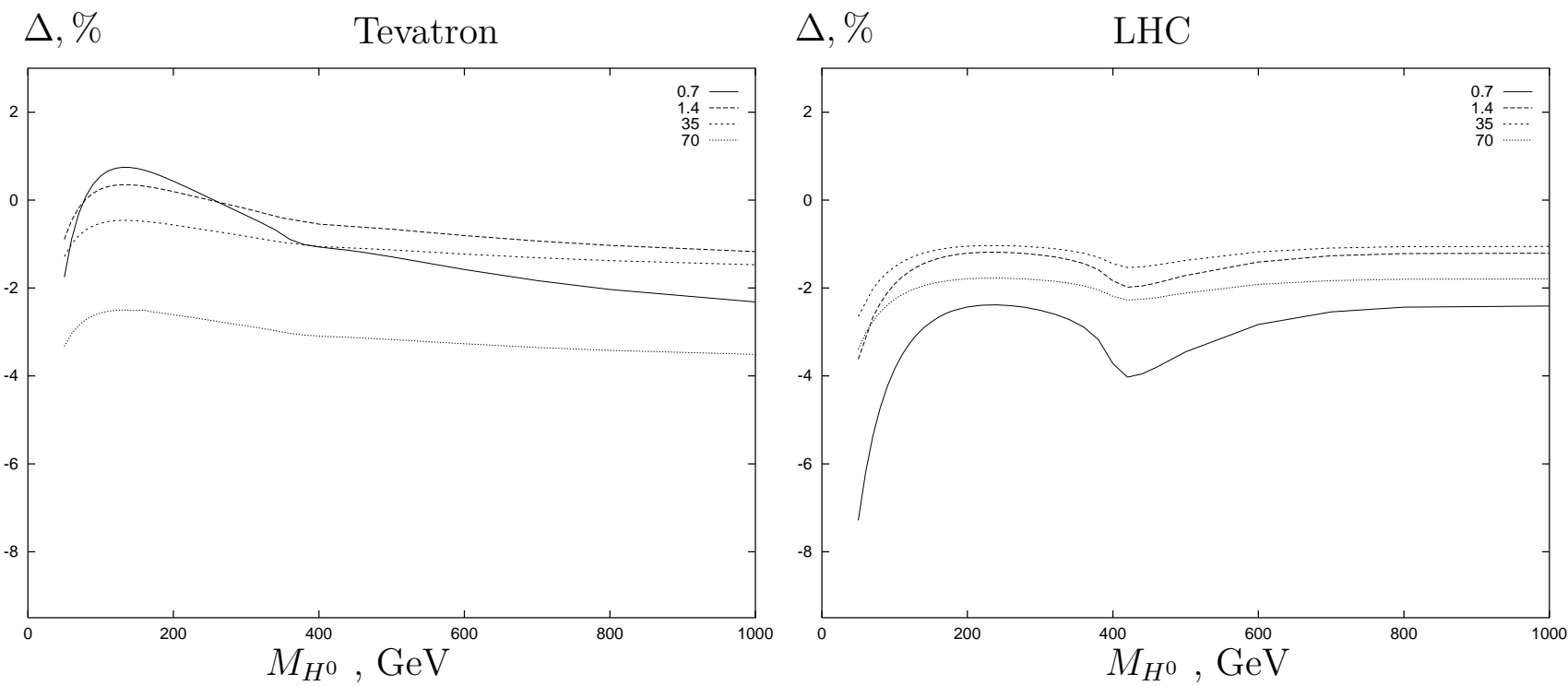

FIG. 11. The variation of the relative correction $\Delta$ with $M_{H^{0}}$ and $\tan \beta$ at the Tevatron $\left(S=(2 \mathrm{TeV})^{2}\right)$ and at the $\operatorname{LHC}\left(S=(14 \mathrm{TeV})^{2}\right)$ within the G2HDM (with $M_{h^{0}}=45 \mathrm{GeV}$, $M_{A^{0}}=50 \mathrm{GeV}, M_{H^{ \pm}}=50 \mathrm{GeV}$ and $\left.\alpha=\pi / 2\right)$.

$B_{1}$ functions depending on the choice of the $L, R$-mixing angle $\Phi_{\tilde{t}}$ (see also [20] (Tevatron)). Finally, we show in the Figs. 16,17,18 the impact of the complete $\mathcal{O}(\alpha)$ contribution within the MSSM (including the $W, Z$ boson contribution) on the hadronic cross sections for top pair production at the Tevatron and the LHC. There, we imposed additional constraints to account for the experimental bounds on the supersymmetric particle mass spectrum from the negative search at LEP and only allow those parameter combinations which yield

$$
\begin{gathered}
M_{h^{0}}>45 \mathrm{GeV} \\
M_{\tilde{\chi}_{1,2}^{ \pm}}>65 \mathrm{GeV}, M_{\tilde{\chi}^{0}}^{\text {lightest }}>24 \mathrm{GeV}, M_{\tilde{\chi}^{0}}^{\text {next-to-lightest }}>46 \mathrm{GeV} .
\end{gathered}
$$

We already emphasized the most interesting features of the radiative corrections originating from the MSSM Higgs sector and the SUSY EW-like contribution. To summarize we show the dependence of the relative corrections on the MSSM input parameters

$$
\tan \beta, M_{A^{0}}, \mu, M_{2}, m_{\tilde{b}_{1}}, m_{\tilde{t}_{2}}, \Phi_{\tilde{t}}
$$

within the following MSSM scenarios:

- Fig. 16: variation with $M_{A^{0}}$ for different values of $\tan \beta$,

- Fig. 17: variation with $\mu$ for different values of $M_{2}$,

- Fig. 18: variation with $m_{\tilde{t}_{2}}$ for

a.) different values of $\tan \beta$ (with $M_{A^{0}}=450 \mathrm{GeV}, m_{\tilde{b}_{1}}=150 \mathrm{GeV}$ and $\Phi_{\tilde{t}}=\pi / 4$ ),

b.) different values of the $L, R$-mixing angle $\Phi_{\tilde{t}}$ (with $M_{A^{0}}=150 \mathrm{GeV}, \tan \beta=0.7$ and $\left.m_{\tilde{b}_{1}}=500 \mathrm{GeV}\right)$ and c.) different combinations of the values of $\left(m_{\tilde{b}_{1}} ; \Phi_{\tilde{t}}\right)$ (with $M_{A^{0}}=150 \mathrm{GeV}$ and $\left.\tan \beta=0.7\right)$. 

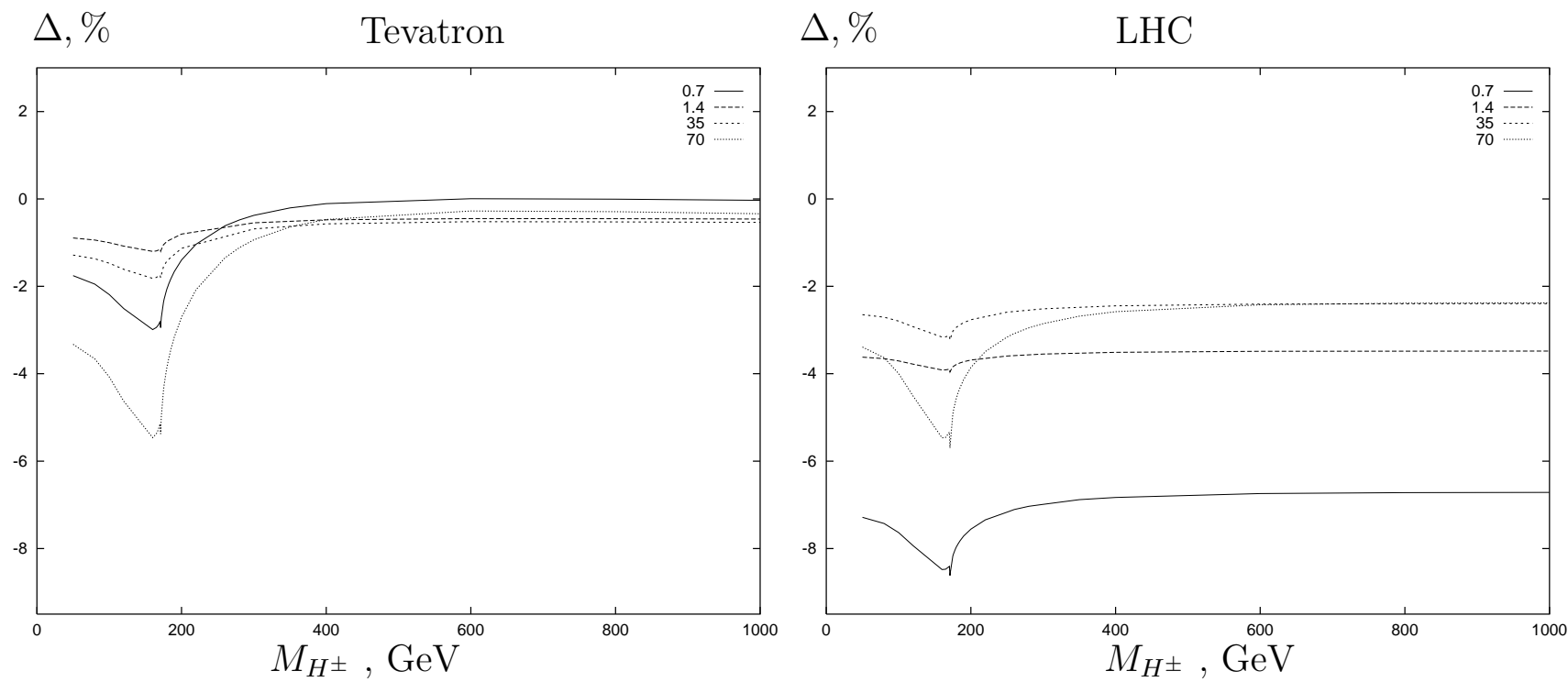

FIG. 12. The variation of the relative correction $\Delta$ with $M_{H^{ \pm}}$and $\tan \beta$ at the Tevatron $\left(S=(2 \mathrm{TeV})^{2}\right)$ and at the LHC $\left(S=(14 \mathrm{TeV})^{2}\right)$ within the G2HDM (with $M_{h^{0}}=45 \mathrm{GeV}$, $M_{A^{0}}=50 \mathrm{GeV}, M_{H^{0}}=50 \mathrm{GeV}$ and $\left.\alpha=\pi / 2\right)$.

It is interesting to compare these effects with the relative corrections obtained when using the best $\chi^{2}$-fit results of a global MSSM-fit to most recent electroweak precision data [32]: $\Delta_{\text {Tevatron }}=-0.7 \% ;-1.6 \%$ and $\Delta_{L H C}=-4.3 \% ;-3.2 \%$ with $\tan \beta=1.6 ; 35$, respectively. As expected the effects are of the same order of magnitude as the one obtained within the MSM. This is due to the decoupling behavior of the MSSM in the limit of very heavy supersymmetric particles which allow for the presence of supersymmetry in electroweak $Z$ pole observables measured at LEP and SLC even though at the present level of accuracy no convincing deviation from the MSM prediction has been observed.

As an interesting alternative to the total top pair production cross section we show in Fig. 19 and Fig. 20 the impact of the G2HDM and the MSSM $\mathcal{O}(\alpha)$ contribution, respectively, on the invariant mass distribution of the produced top quark pair $d \sigma / d M_{t \bar{t}}$

$$
\frac{d \sigma}{d M_{t \bar{t}}}=\sum_{i j=q \bar{q}, g g} \frac{2}{M_{t \bar{t}}} \hat{\sigma}_{i j}(\hat{s}=\tau S) \tau \frac{d L_{i j}}{d \tau}
$$

with $\tau=M_{t \bar{t}}^{2} / S$. There we are especially interested in the distortion of the $t \bar{t}$ invariant mass due to the $s$-channel Higgs-exchange diagrams in the gluon fusion subprocess as a very characteristic signature of the electroweak symmetry breaking sector possibly observable at $p p$ colliders. As can be seen in Figs. 19,20 at the LHC a significant distortion arises for Higgs-boson masses $M_{H^{0}}>2 m_{t}$ for a sufficiently small Higgs-decay width $\Gamma_{H^{0}}$.

\section{CONCLUSIONS}

We have studied the (virtual) effects of the complete $\mathcal{O}(\alpha)$ contribution within the G2HDM and the MSSM to the main top pair production mechanisms, $q \bar{q}$ annihilation and gluon fusion, at future hadron colliders: the upgraded Tevatron with $\sqrt{S}=2 \mathrm{TeV}$

and the LHC with $\sqrt{S}=14 \mathrm{TeV}$. Typically the Born-cross sections are reduced by the order of several percent but in exceptional regions of the parameter space, that is when 

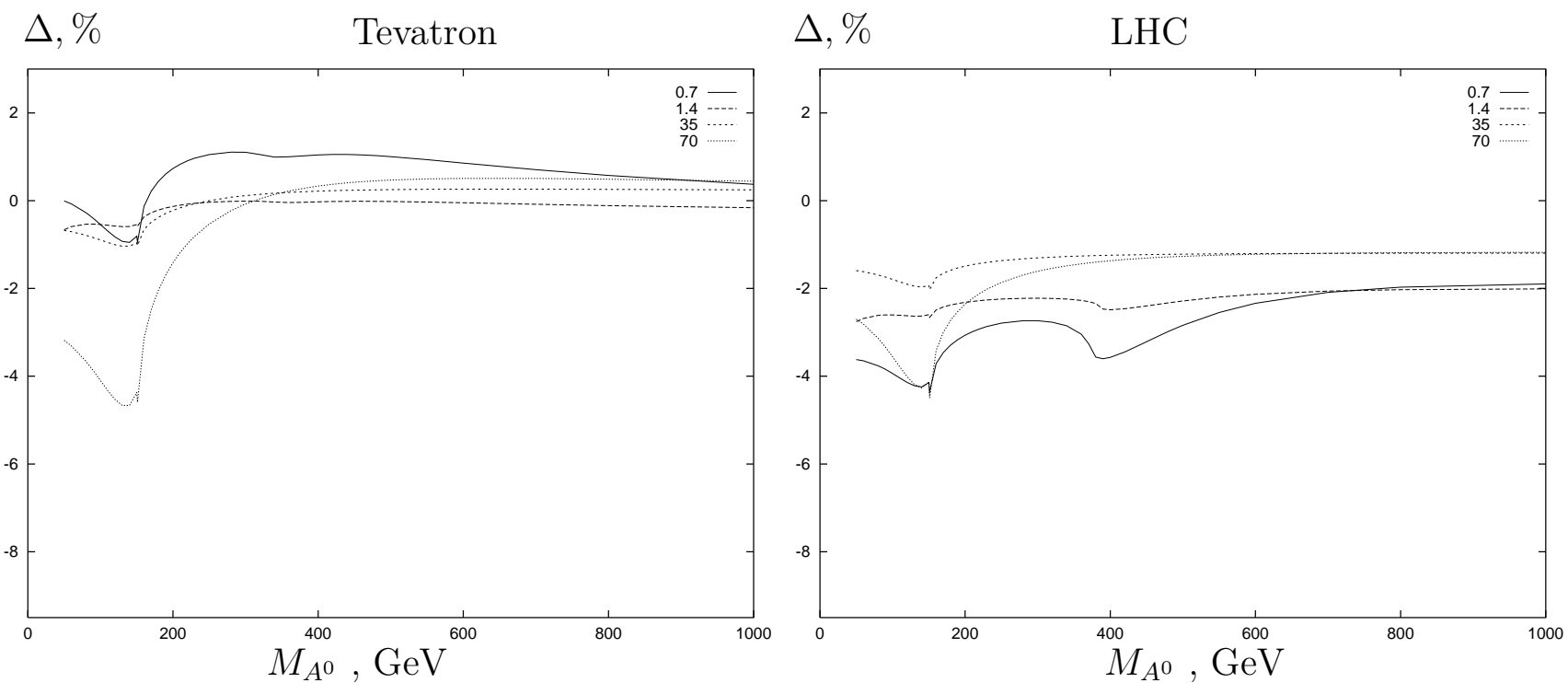

FIG. 13. The variation of the relative correction $\Delta$ with $M_{A^{0}}$ and $\tan \beta$ at the Tevatron and at the LHC within the supersymmetric 2-Higgs-Doublet Model $\left(m_{\tilde{t}_{2}}=75 \mathrm{GeV}, m_{\tilde{b}_{1}}=150 \mathrm{GeV}\right.$, $\Phi_{\tilde{t}}=\pi / 4$ and $\left.\mu=100 \mathrm{GeV}\right)$.

$m_{t} \approx m_{\tilde{t}_{2}}+M_{\tilde{\chi}^{0}} ; m_{b}+M_{H^{ \pm}}$, the radiative corrections can be considerably enhanced: up to about $40 \%$ and $30 \%$ at the upgraded Tevatron and the LHC, respectively. In the gluon fusion subprocess an interesting Higgs-specific signature occurs when due to the $s$-channel Higgs-exchange diagrams the $t \bar{t}$ invariant mass distribution at the LHC can be significantly distorted for Higgs-boson masses $M_{H^{0}}>2 m_{t}$ and a sufficiently small Higgs-decay width $\Gamma_{H^{0}}$. We conclude that provided the intrinsic QCD uncertainties can be considerably reduced there is potential for electroweak precision studies in strong processes at future hadron colliders.

\section{ACKNOWLEDGMENTS}

W.M. M. gratefully acknowledges the opportunity to participate in the summer visitors program of the Theoretical Physics Department of FNAL and the kind hospitality extended to him during the visit when parts of this work were done.

The Fermi National Accelerator Laboratory is operated by the Universities Research Association, Inc., under contract DE-AC02-76CHO3000 with the United States Department of Energy. 

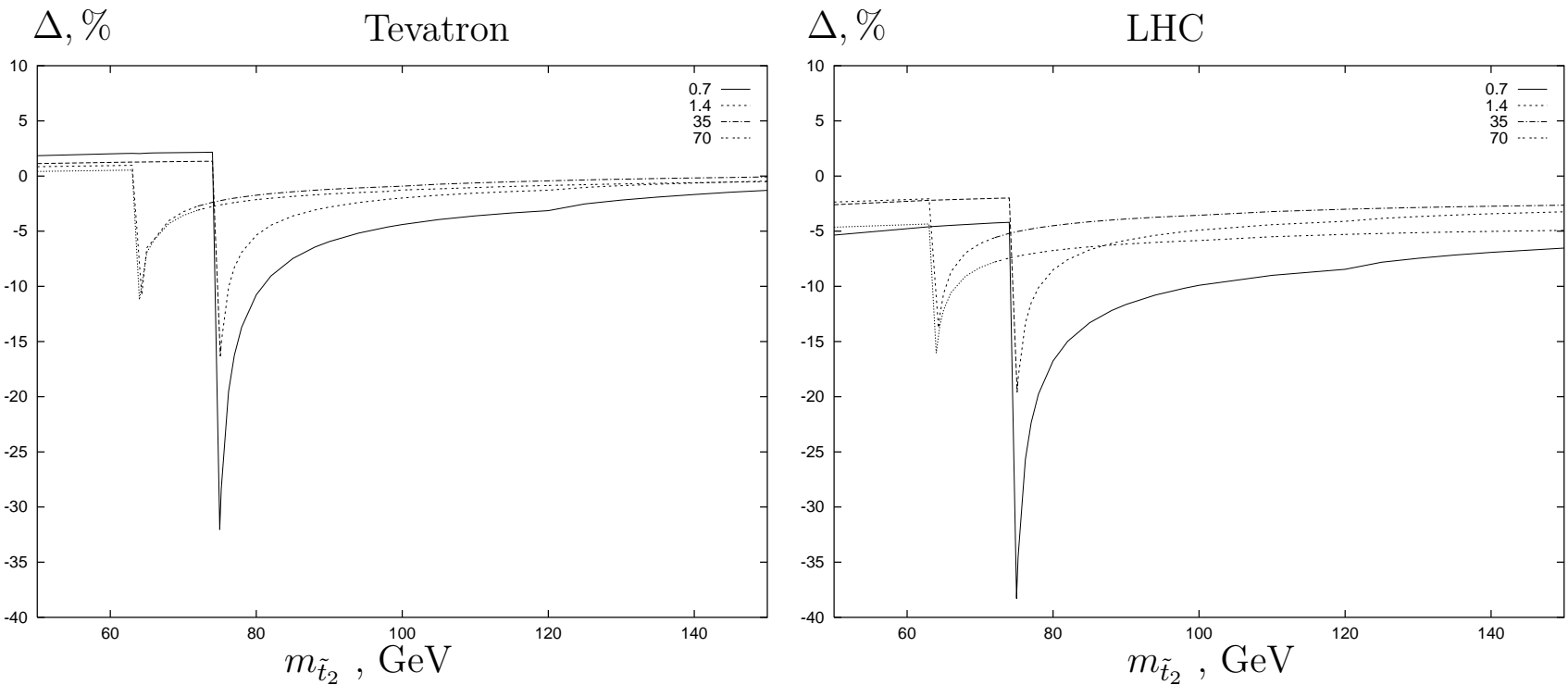

FIG. 14. The variation of the relative correction $\Delta$ with $m_{\tilde{t}_{2}}$ and $\tan \beta$ at the Tevatron and at the LHC when including only the SUSY EW-like one-loop corrections (with $m_{\tilde{b}_{1}}=150 \mathrm{GeV}$, $\Phi_{\tilde{t}}=\pi / 4, \mu=100 \mathrm{GeV}$ and $\left.M_{2}=3|\mu|\right)$.

\section{REFERENCES}

[1] F. Abe et al. (CDF collaboration), Phys. Rev. Lett. 74, 2626 (1995).

[2] S. Abachi et al. (DØ collaboration), Phys. Rev. Lett. 74, 2632 (1995).

[3] A. Salam, in Elementary Particle Theory, 8th Nobelsymposium, Wiley N.Y. (1969); S. Weinberg, Phys. Rev. Lett. 19, 1264 (1967); S.L. Glashow, Nucl. Phys. 22, 579 (1961); S.L. Glashow, J. Iliopoulos and L. Maiani, Phys. Rev. D2, 1285 (1970).

[4] P.W. Higgs, Phys. Lett. 12, 131 (1964), Phys. Rev. Lett. 13, 508 (1964) and Phys. Rev. Lett. 145, 1156 (1966); T.W.B. Kibble, Phys. Rev. 155, 1554 (1967); R. Brout and F. Englert, Phys. Rev. Lett. 13, 321 (1964).

[5] P.C. Bhat, for the $\mathrm{D} \varnothing$ collaboration, talk presented at the Wine and Cheese Seminar at Fermilab, February 1997.

[6] The LEP Electroweak Working Group and the SLD Heavy Flavour Group, LEPEWWG/97-01, April 1997.

[7] D.S. Kestenbaum, for the CDF collaboration, talk presented at the 16th International Conference on Physics in Collision (PIC96), Mexico City, June 1996, published in the proceedings, Fermilab-Conf-97/016-E.

[8] E.L. Berger and H. Contopaganos, Phys. Rev. D54, 3085 (1996).

[9] S. Catani, M.L. Mangano, P. Nason and L. Trentadue, Phys. Lett. B378, 329 (1996).

[10] E. Laenen, J. Smith and W.L. van Neerven, Nucl. Phys. B369, 543 (1992) and Phys. Lett. B321, 254 (1994).

[11] W. Beenakker, A. Denner, W. Hollik, R. Mertig, T. Sack and D. Wackeroth, Nucl. Phys. B411, 343 (1994).

[12] Report of the tev2000 Study Group, D. Amedei and R. Brock (eds.), FERMILAB-Pub96/082, April 1996.

[13] J.F. Gunion, H.E. Haber, G.L. Kane and S. Dawson, The Higgs Hunter's Guide, Addison-Wesley Reading, MA (1990) (Erratum: hep-ph/9302272, February 1993).

[14] For a review see, e.g. , 30] and J. Wess and J. Bagger, Supersymmetry and Supergravity, 

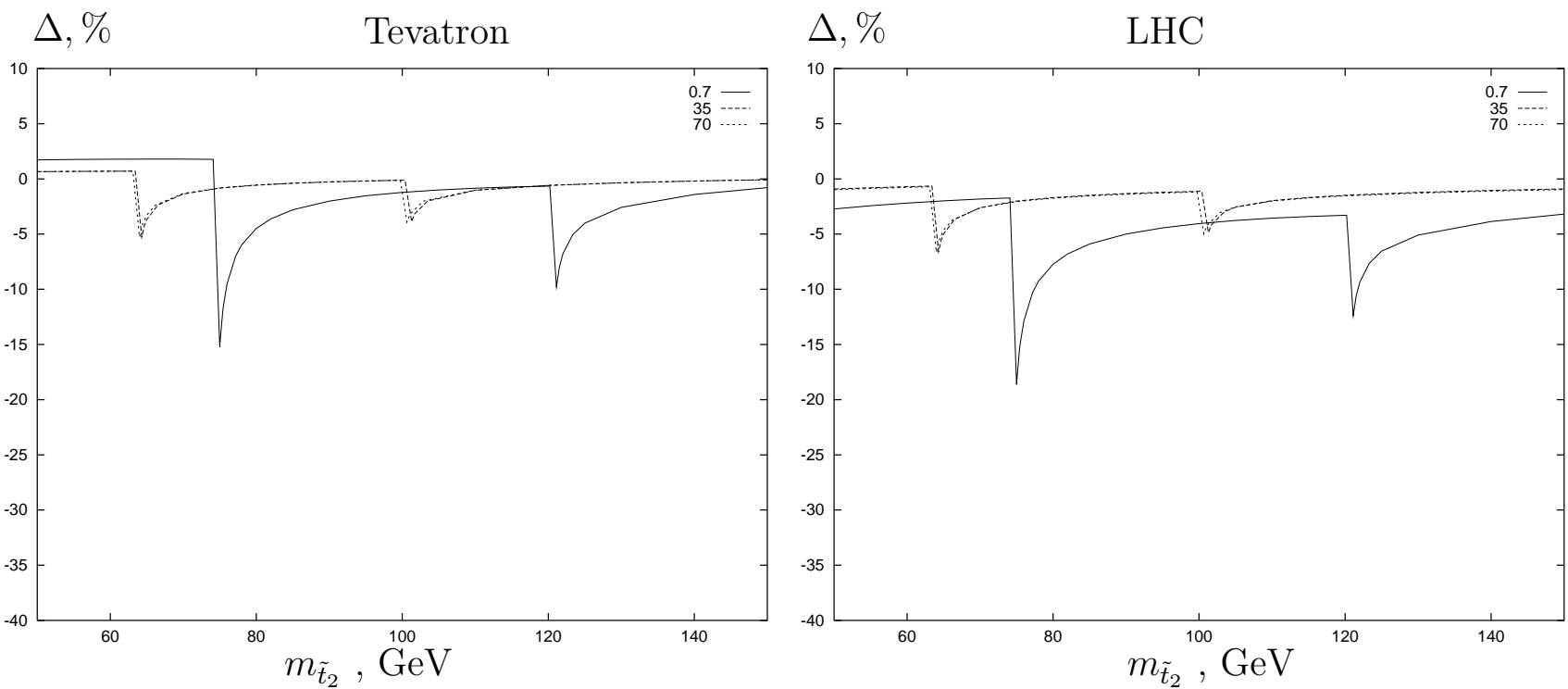

FIG. 15. The variation of the relative correction $\Delta$ with $m_{\tilde{t}_{2}}$ and $\tan \beta$ at the Tevatron and at the LHC when including only the SUSY EW-like one-loop corrections (with $\mu=100 \mathrm{GeV}$, $M_{2}=3|\mu|, m_{\tilde{b}_{1}}=1 \mathrm{TeV}$ and $\left.\Phi_{\tilde{t}}=0\right)$.

Princeton University Press 1983; P. Fayet and S. Ferrara, Phys. Rep. 32, 249 (1977) and H.-P. Nilles, Phys. Rep. 110, 1 (1984).

[15] C. Kao, G.A. Ladinsky and C.P. Yuan, FSU-HEP-940508, June 1994, published in the DPF94 Conference proceedings.

[16] A. Stange and S. Willenbrock, Phys. Rev. D49, 1354 (1994).

[17] H.Y. Zhou, C.S. Li and Y.P. Kuang, Phys. Rev. D55, 4412 (1997).

[18] D. Wackeroth, Diploma Thesis, Technische Universität München, April 1992.

[19] S. Alam, K. Hagiwara and S. Matsumoto, Phys. Rev. D55, 1307 (1997); Z. Sullivan, hep-ph/9611302, November 1996, to be published in Phys.Rev.D; C.S. Li, H.Y. Zhou, Y.L. Zhu and J.M. Yang, Phys. Lett. B379, 135 (1996); C.S. Li, B.Q. Hu, J.M. Yang and C.G. Hu, Phys. Rev. D52, 5014 (1995) (Erratum: Phys. Rev. D53, 4112(E) (1996)).

[20] J. Kim, J.L. Lopez, D.V. Nanopoulos and R. Rangarajan, Phys. Rev. D54, 4364 (1996).

[21] H.Y. Zhou and C.S. Li, Phys. Rev. D55, 4421 (1997).

[22] J.M. Yang and C.S. Li, Phys. Rev. D54, 4380 (1996) (Erratum: Phys. Rev. D54, 3671(E) (1996)).

[23] J.P. Martin, talk presented at the ICHEP96, Warsaw, 25-31 July 1996, to appear in the proceedings.

[24] B.W. Lee, C. Quigg and H.B. Thacker, Phys. Rev. D16, 1519 (1977).

[25] The Particle Data Group, Phys. Rev. D54, no.1 (1996).

[26] M. Demarteau, talk presented at the DPF96 Conference, Minneapolis, August 1996, to appear in the proceedings.

[27] J. Ellis, G. Ridolfi, F. Zwirner, Phys. Lett. B262, 477 (1991) and Phys. Lett. B257, 83 (1991).

[28] M.A. Diaz and H.E. Haber, Phys. Rev. D45, 4246 (1992).

[29] J.F. Gunion and H.E. Haber, Nucl. Phys. B272, 1 (1986), Nucl. Phys. B278, 449 (1986) and Nucl. Phys. B307, 445 (1988) (Errata: hep-ph/9301205, January 1993).

[30] H.E. Haber and G.L. Kane, Phys. Rep. 117,75 (1985). 


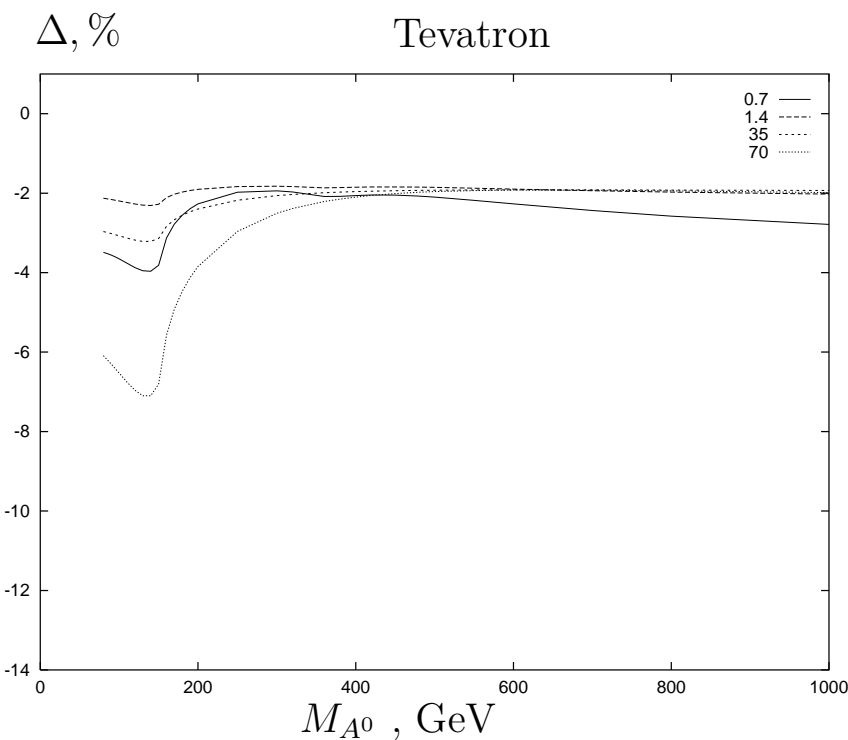

$\Delta, \% \quad$ LHC

FIG. 16. The variation of the relative correction $\Delta$ with $M_{A^{0}}$ and $\tan \beta$ at the Tevatron and at the LHC within the MSSM (with $m_{\tilde{t}_{2}}=75 \mathrm{GeV}, \mu=-120 \mathrm{GeV}, M_{2}=3|\mu|, m_{\tilde{b}_{1}}=150 \mathrm{GeV}$ and $\left.\Phi_{\tilde{t}}=\pi / 4\right)$.

[31] A.D. Martin, R.G. Roberts and W.J. Stirling, Phys. Rev. D50, 6734 (1994).

[32] W.de Boer, A. Dabelstein, W. Hollik, W.M. Mösle and U. Schwickerath, IEKP-KA/9608, hep-ph/9609209, November 1996, to be published in Phys.Rev.D. 

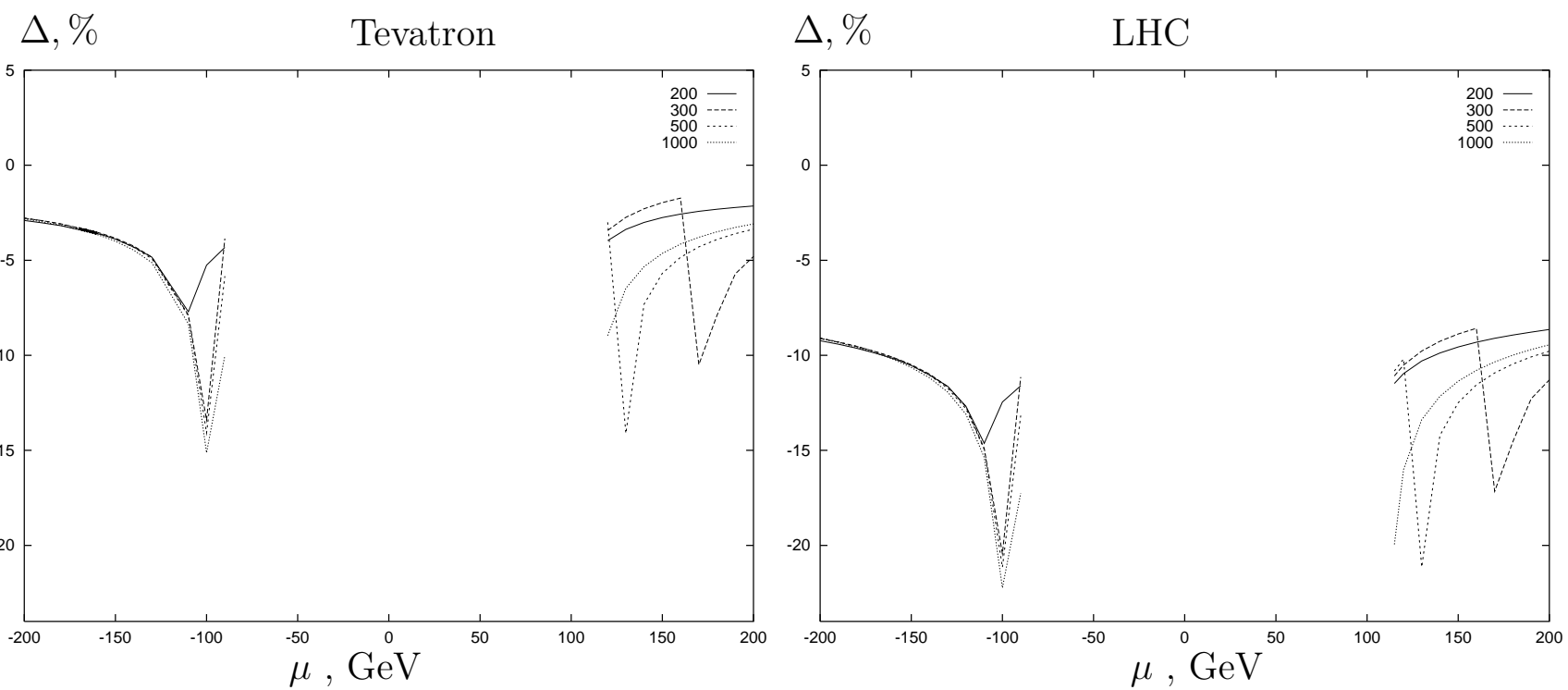

FIG. 17. The variation of the relative correction $\Delta$ with $\mu$ and $M_{2}$ at the Tevatron and at the LHC within the MSSM (with $M_{A^{0}}=150 \mathrm{GeV}, m_{\tilde{t}_{2}}=75 \mathrm{GeV}, m_{\tilde{b}_{1}}=1 \mathrm{TeV}, \Phi_{\tilde{t}}=0$ and $\tan \beta=0.7)$. 
$\Delta, \%$ Tevatron
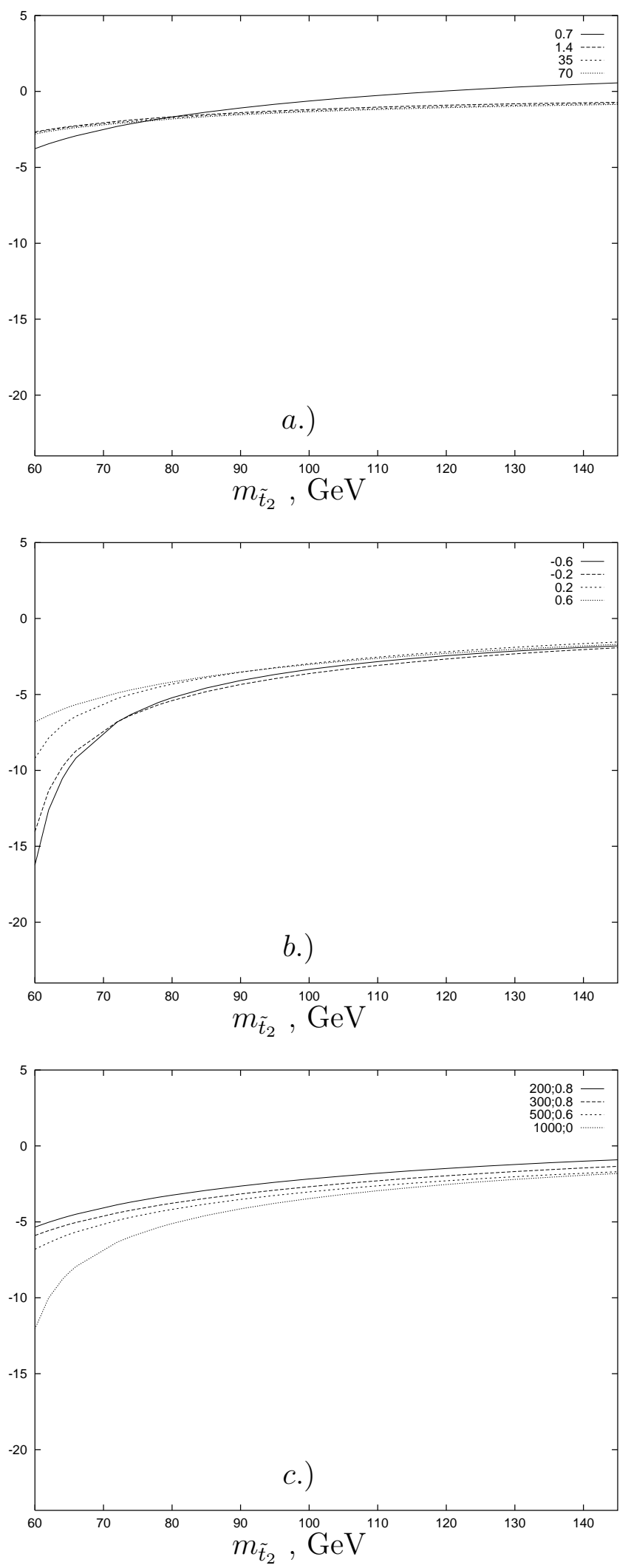

$\Delta, \% \mathrm{LHC}$
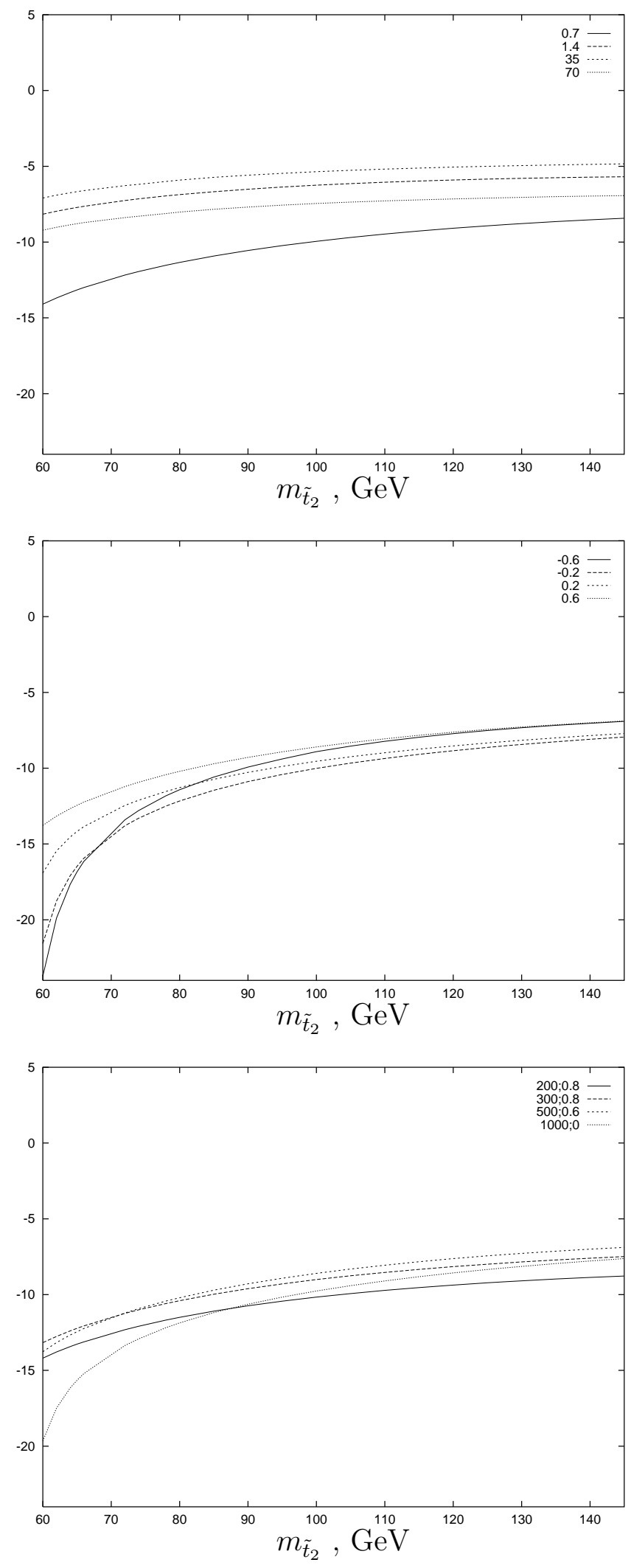

FIG. 18. The variation of the relative correction $\Delta$ with $m_{\tilde{t}_{2}}$ at the Tevatron and at the LHC for different MSSM scenarios (with $\mu=-120 \mathrm{GeV}$ and $M_{2}=3|\mu|$ ). 

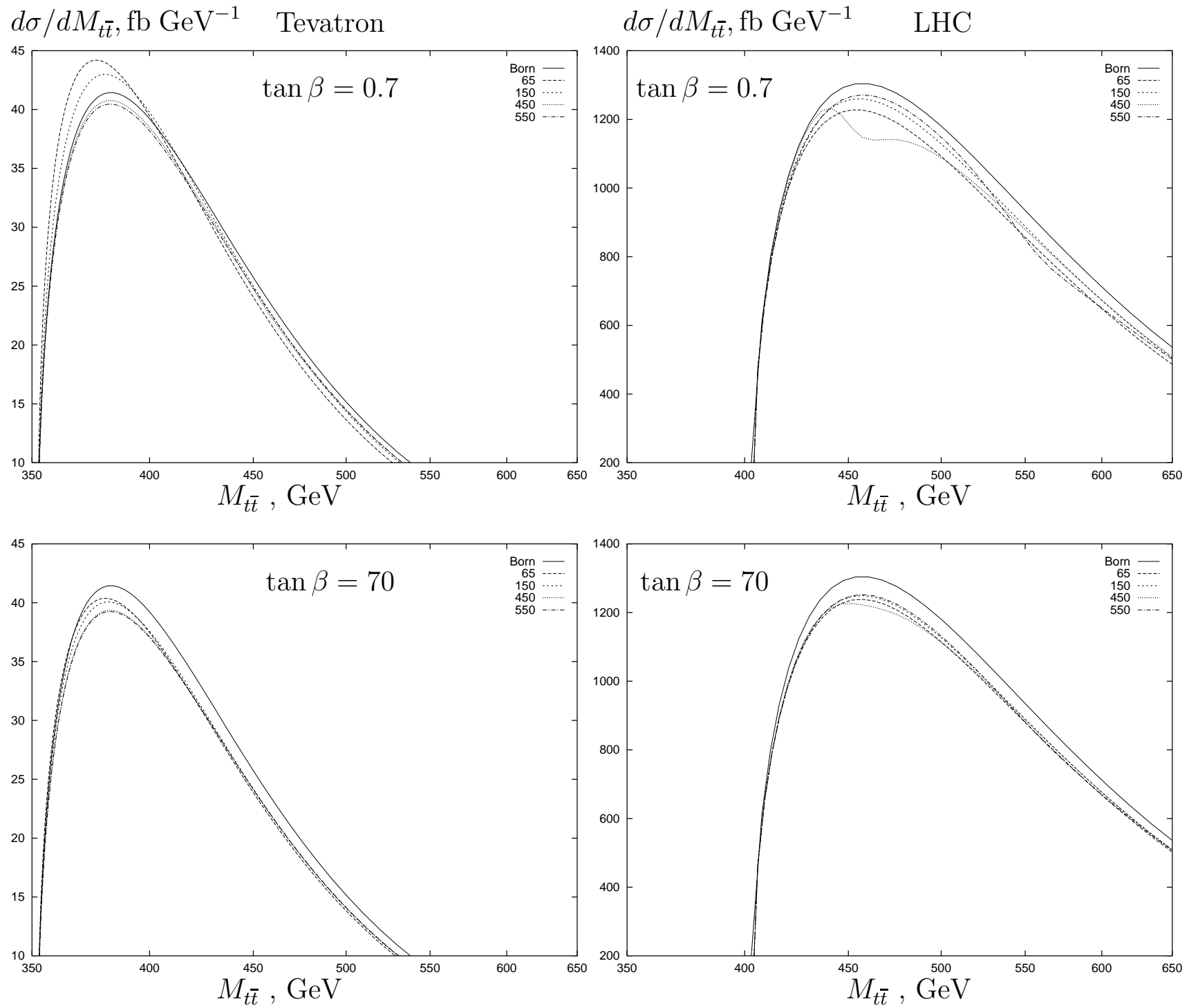

FIG. 19. The invariant mass $M_{t \bar{t}}$ distribution within the G2HDM for different values of $M_{H^{0}}$ and $\tan \beta$ (with $M_{h^{0}}=45 \mathrm{GeV}, M_{A^{0}}=50 \mathrm{GeV}, M_{H^{ \pm}}=150 \mathrm{GeV}$ and $\alpha=\pi / 2$ ). 

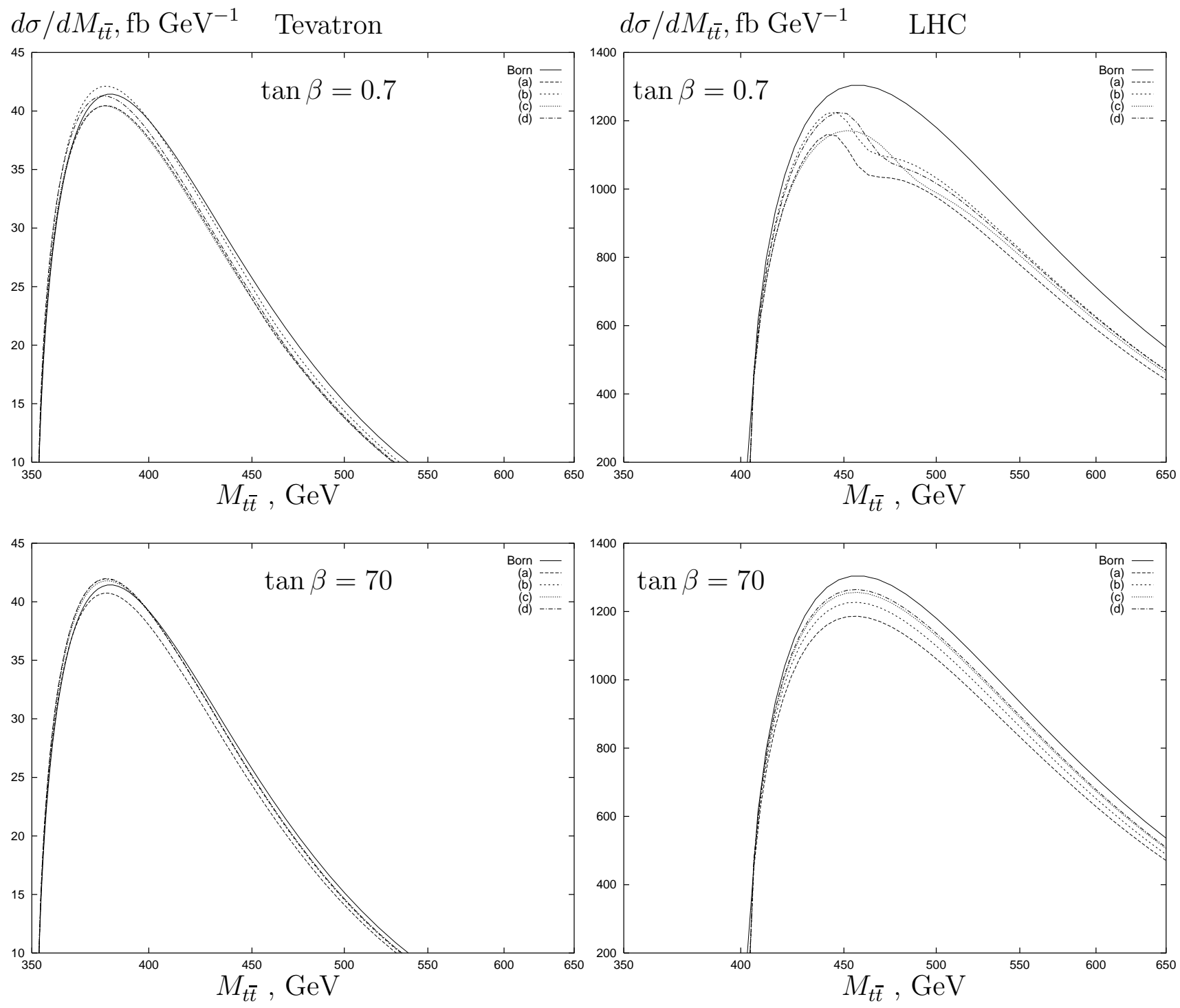

FIG. 20. The invariant mass $M_{t \bar{t}}$ distribution within different MSSM scenarios (with $M_{A^{0}}=450$ $\mathrm{GeV}, M_{2}=3|\mu|$ and with (a) $m_{\tilde{t}_{2}}=50, m_{\tilde{b}_{1}}=150 \mathrm{GeV}, \Phi_{\tilde{t}}=\pi / 4, \mu=-120 \mathrm{GeV}$, (b) same as (a) but with $m_{\tilde{t}_{2}}=75 \mathrm{GeV}$, (c) $m_{\tilde{t}_{2}}=75, m_{\tilde{b}_{1}}=400 \mathrm{GeV}, \Phi_{\tilde{t}}=-\pi / 4, \mu=150 \mathrm{GeV}$ and (d) $\left.m_{\tilde{t}_{2}}=75, m_{\tilde{b}_{1}}=800 \mathrm{GeV}, \Phi_{\tilde{t}}=0, \mu=150 \mathrm{GeV}\right)$. 